\title{
Shrinkage Priors for Bayesian Penalized Regression.
}

\author{
Sara van $\operatorname{Erp}^{1}$, Daniel L. Oberski ${ }^{2}$, and Joris Mulder ${ }^{1}$ \\ ${ }^{1}$ Tilburg University \\ ${ }^{2}$ Utrecht University
}

This manuscript has been published in the Journal of Mathematical Psychology. Please cite the published version:

Van Erp, S., Oberski, D. L., \& Mulder, J. (2019). Shrinkage priors for Bayesian penalized regression. Journal of Mathematical Psychology, 89, 31-50.

doi:10.1016/j.jmp.2018.12.004 


\begin{abstract}
In linear regression problems with many predictors, penalized regression techniques are often used to guard against overfitting and to select variables relevant for predicting an outcome variable. Recently, Bayesian penalization is becoming increasingly popular in which the prior distribution performs a function similar to that of the penalty term in classical penalization. Specifically, the so-called shrinkage priors in Bayesian penalization aim to shrink small effects to zero while maintaining true large effects. Compared to classical penalization techniques, Bayesian penalization techniques perform similarly or sometimes even better, and they offer additional advantages such as readily available uncertainty estimates, automatic estimation of the penalty parameter, and more flexibility in terms of penalties that can be considered. However, many different shrinkage priors exist and the available, often quite technical, literature primarily focuses on presenting one shrinkage prior and often provides comparisons with only one or two other shrinkage priors. This can make it difficult for researchers to navigate through the many prior options and choose a shrinkage prior for the problem at hand. Therefore, the aim of this paper is to provide a comprehensive overview of the literature on Bayesian penalization. We provide a theoretical and conceptual comparison of nine different shrinkage priors and parametrize the priors, if possible, in terms of scale mixture of normal distributions to facilitate comparisons. We illustrate different characteristics and behaviors of the shrinkage priors and compare their performance in terms of prediction and variable selection in a simulation study. Additionally, we provide two empirical examples to illustrate the application of Bayesian penalization. Finally, an $\mathrm{R}$ package bayesreg is available online (https://github.com/sara-vanerp/bayesreg) which allows researchers to perform Bayesian penalized regression with novel shrinkage priors in an easy manner.
\end{abstract}

Bayesian, Shrinkage Priors, Penalization, Empirical Bayes, Regression 


\section{BAyesian Penalization}

\section{Introduction}

Regression analysis is one of the main statistical techniques often used in the field of psychology to determine the effect of a set of predictors on an outcome variable. The number of predictors is often large, especially in the current "Age of Big Data". For example, the Kavli HUMAN project (Azmak et al., 2015) aims to collect longitudinal data on all aspects of human life for 10,000 individuals. Measurements include psychological assessments (e.g., personality, IQ), health assessments (e.g., genome sequencing, brain activity scanning), social network assessment, and variables related to education, employment, and financial status, resulting in an extremely large set of variables. Furthermore, personal tracking devices allow the collection of large amounts of data on various topics, including for example mood, in a longitudinal manner (Fawcett, 2015). The problem with regular regression techniques such as ordinary least squares (OLS) is that they quickly lead to overfitting as the ratio of predictor variables to observations increases (see for example, McNeish, 2015, for an overview of the problems with OLS).

Penalized regression is a statistical technique widely used to guard against overfitting in the case of many predictors. Penalized regression techniques have the ability to select variables out of a large set of variables that are relevant for predicting some outcome. Therefore, a popular setting for penalized regression is in high-dimensional data, where the number of predictors $p$ is larger than the sample size $n$. Furthermore, in settings where the number of predictors $p$ is smaller than the sample size $n$ (but still relatively large), penalized regression can offer advantages in terms of avoiding overfitting and achieving model parsimony compared to traditional variable selection methods such as null-hypothesis testing or stepwise selection methods (Derksen and Keselman, 1992; Tibshirani, 1996). The central idea of penalized regression approaches is to add a penalty term to the minimization of the sum of squared residuals, with the goal of shrinking small coefficients towards zero while leaving large coefficients large, i.e.,

$$
\begin{gathered}
\underset{\beta_{0}, \boldsymbol{\beta}}{\operatorname{minimize}}\left\{\frac{1}{2 n}\left\|\boldsymbol{y}-\beta_{0} \mathbf{1}-\boldsymbol{X} \boldsymbol{\beta}\right\|_{2}^{2}+\lambda_{c}\|\boldsymbol{\beta}\|_{q}\right\}, \\
\text { where }\|\boldsymbol{\beta}\|_{q}=\left(\sum_{j=1}^{p}\left|\beta_{j}\right|^{q}\right)^{\frac{1}{q}},
\end{gathered}
$$

where $\boldsymbol{y}=\left(y_{1}, \ldots, y_{n}\right)^{\prime}$ is an $n$-dimensional vector containing the observations on the outcome variable, $\beta_{0}$ reflects the intercept, 1 is an $n$-dimensional 


\section{BAYESIAN PENALIZATION}

vector of ones, $\boldsymbol{X}$ is an $(n \times p)$ matrix of the observed scores on the $p$ predictor variables, and $\boldsymbol{\beta}=\left(\beta_{1}, \ldots, \beta_{p}\right)^{\prime}$ is a $p$-dimensional parameter vector of regression coefficients. $\lambda_{c}$ reflects the penalty parameter, with large values resulting in more shrinkage towards zero while $\lambda_{c}=0$ leads to the ordinary least squares solution. The choice of $q$ determines the type of penalty induced, for example, $q=1$ results in the well-known least absolute shrinkage and selection operator (lasso; Tibshirani, 1996) solution and $q=2$ results in the ridge solution (Hoerl and Kennard, 1970). We refer to Hastie et al. (2015) for a comprehensive introduction and overview of various penalized regression methods in a frequentist framework.

It is well known that many solutions to the penalized minimization problem in Equation (1) can also be obtained in the Bayesian framework by using a specific prior combined with the posterior mode estimate, which has been shown to perform similar to or better than their classical counterparts (Hans, 2009; Kyung et al., 2010; Li and Lin, 2010). ${ }^{1}$ Adopting a Bayesian perspective on penalized regression offers several advantages. First, penalization fits naturally in a Bayesian framework since a prior distribution is needed anyway and shrinkage towards zero can be straightforwardly achieved by choosing a specific parametric form for the prior. Second, parameter uncertainty and standard errors follow naturally from the posterior standard deviations. As shown by Kyung et al. (2010) classical penalized regression procedures can result in estimated standard errors that suffer from multiple problems, such as variances estimated to be 0 (in the case of sandwich estimates), and unstable or poorly performing variance estimates (in the case of bootstrap estimates). Third, with Bayesian penalization it is possible to estimate the penalty parameter(s) $\lambda$ simultaneously with the model parameters in a single step. This is especially advantageous when there are multiple penalty parameters (e.g., in the elastic net; Zou and Hastie, 2005), since sequential cross-validation procedures to determine multiple penalty parameters induce too much shrinkage (i.e., the double shrinkage problem; see e.g., Zou and Hastie, 2005). Fourth, Bayesian penalization relies on Markov Chain Monte Carlo (MCMC) sampling rather than optimization, which provides more flexibility in the sense that priors that would correspond to non-convex penalties (i.e., $q<1$ in (1)) are easier to implement. Non-convex penalties would result in multiple modes, making them difficult to implement in an optimization framework. The cost of the flexibility of MCMC, however, is that it requires more computation time compared to standard optimization procedures. Finally, Bayesian estimates have an intuitive interpretation. For example, a

\footnotetext{
${ }^{1}$ Note that from a Bayesian perspective, however, there is no theoretical justification for reporting the posterior mode estimate (Tibshirani, 2011).
} 


\section{BAyESian PENALIZATION}

95\% Bayesian credibility interval can simply be interpreted as the interval in which the true value lies with $95 \%$ probability (e.g., Berger, 2006).

Due to these advantages, Bayesian penalization is becoming increasingly popular in the literature (see e.g., Alhamzawi et al., 2012; Andersen et al., 2017; Armagan et al., 2013; Bae and Mallick, 2004; Bhadra et al., 2016; Bhattacharya et al., 2012; Bornn et al., 2010; Caron and Doucet, 2008; Carvalho et al., 2010; Feng et al., 2017; Griffin and Brown, 2017; Hans, 2009; Ishwaran and Rao, 2005; Lu et al., 2016; Peltola et al., 2014; Polson and Scott, 2011; Roy and Chakraborty, 2016; Zhao et al., 2016). An active area of research investigates theoretical properties of priors for Bayesian penalization, such as the Bayesian lasso prior (for a recent overview, see Bhadra et al., 2017). In addition to the Bayesian counterparts of classical penalized regression solutions, many other priors have been proposed that have desirable properties in terms of prediction and variable selection. However, the extensive (and often technical) literature and subtle differences between the priors can make it difficult for researchers to navigate the options and make sensible choices for the problem at hand. Therefore, the aim of this paper is to provide a comprehensive overview of the priors that have been proposed for penalization in (sparse) regression. We use the term shrinkage priors to emphasize that these priors aim to shrink small effects towards zero. We place the shrinkage priors in a general framework of scale mixtures of normal distributions to emphasize the similarities and differences between the priors. By providing insight in the characteristics and behaviors of the priors, we aid researchers in choosing a prior for their specific problem. Additionally, we present a straightforward method to obtain empirical Bayes (EB) priors for Bayesian penalization. We conduct a simulation study to compare the performance of the priors in terms of prediction and variable selection in a linear regression model, and provide two empirical examples to further illustrate the Bayesian penalization methods. Finally, the shrinkage priors have been implemented in the $\mathrm{R}$ package bayesreg, available from https://github.com/sara-vanerp/bayesreg, to allow general utilization.

The remainder of this paper is organized as follows: Section 2 introduces Bayesian penalized regression. A theoretical overview of the different shrinkage priors can be found in Section 3. Further insights into the priors is provided through illustrations in Section 4 and the priors are compared in a simulation study in Section 5. Section 6 presents the empirical applications, followed by a discussion in Section 7 . 


\section{Bayesian penalized regression}

The likelihood for the linear regression model is given by:

$$
y_{i} \mid \beta_{0}, \boldsymbol{x}_{i}, \beta, \sigma^{2} \sim \operatorname{Normal}\left(\beta_{0}+\sum_{j=1}^{p} x_{i j} \beta_{j}, \sigma^{2}\right),
$$

where $\beta_{0}$ represents the intercept, $\beta_{j}$ the regression coefficient for predictor $j$, and $\sigma^{2}$ is the residual variance.

In a Bayesian analysis, a prior distribution is specified for each parameter in the model, e.g., $p\left(\beta_{0}, \boldsymbol{\beta}, \sigma^{2}, \lambda\right)=p\left(\beta_{0}\right) p\left(\boldsymbol{\beta} \mid \sigma^{2}, \lambda\right) p\left(\sigma^{2}\right) p(\lambda)$. Note that the prior for $\beta$ is conditioned on the residual variance $\sigma^{2}$, as well as on $\lambda$. The conditioning on $\sigma^{2}$ is necessary in certain cases to obtain a unimodal posterior (Park and Casella, 2008). In Bayesian penalized regression, $\lambda$ is a parameter in the prior (i.e., a hyperparameter) but has a similar role as the penalty parameter in classical penalized regression. Since this penalty parameter $\lambda$ is used to penalize the regression coefficient, it only appears in the prior for $\beta$. Throughout this paper we will focus on priors for the regression coefficients $\beta_{1}, \ldots, \beta_{j}$ and we will assume noninformative improper priors for the nuisance parameters, specifically, $p\left(\beta_{0}\right)=1$ and a uniform prior on $\log \left(\sigma^{2}\right)$, i.e., $p\left(\sigma^{2}\right)=\sigma^{-2}$. Please note that these priors are chosen as noninformative choices for the linear regression model considered in this paper. However, other choices (including informative priors when prior information is available) are possible and might be preferred in other applications. We refer the reader to van Erp et al. (2018) for general recommendations on specifying prior distributions. We generally assume that the priors for the regression coefficients are independent, unless stated otherwise.

The prior distribution is then multiplied by the likelihood of the data to obtain the posterior distribution, i.e.,

$$
p\left(\beta_{0}, \boldsymbol{\beta}, \sigma^{2}, \lambda \mid \boldsymbol{y}, X\right) \propto p\left(\boldsymbol{y} \mid X, \beta_{0}, \boldsymbol{\beta}, \sigma^{2}\right) p\left(\beta_{0}\right) p\left(\boldsymbol{\beta} \mid \sigma^{2}, \lambda\right) p\left(\sigma^{2}\right) p(\lambda) .
$$

Here, the normalizing constant is not included such that the right-hand side is proportional to the posterior. The only difference with the unpenalized problem (e.g., Bayesian linear regression) is the introduction of the penalty parameter $\lambda$. As a result of the shrinkage prior the posterior in (3) is generally more concentrated, or "shrunk towards", zero in comparison to the likelihood of the model.

An important choice is how to specify the penalty parameter $\lambda$. There are different possibilities for this.

1. Full Bayes. Treat $\lambda$ as an unknown model parameter for which a prior needs to be specified. Typically, a vague prior $p(\lambda)$ is specified for 


\section{BAYESian Penalization}

$\lambda$. Due to its similarity with multilevel (or hierarchical) modeling, full Bayes (FB) is also known as "hierarchical Bayes" (see e.g., Wolpert and Strauss, 1996). This results in a fully Bayesian solution that incorporates the uncertainty about $\lambda$. The advantage of this approach is that the model can be estimated in one step. Throughout this paper, we will consider the half-Cauchy prior on $\lambda$, i.e., $\lambda \sim \operatorname{half-Cauchy}(0,1)$, which is a robust alternative and a popular prior distribution in the Bayesian literature (see e.g., Gelman, 2006; Mulder and Pericchi, 2018; Polson and Scott, 2012).

2. Empirical Bayes. Empirical Bayes (EB) methods, also known as the "evidence" procedure (see e.g., Wolpert and Strauss, 1996), first estimate the penalty parameter $\lambda$ from the data and then plug in this EB estimate for $\lambda$ in the model (see van de Wiel et al., 2017, for an overview of EB methodology in high-dimensional data). The resulting prior is called an EB prior. Since an EB estimate is used for $\lambda$, the EB approach does not require the specification of a prior $p(\lambda)$ as in the FB approach. Since the exact choice of this prior can sometimes have a serious effect on the Bayesian estimates (Roy and Chakraborty, 2016), the EB approach would avoid sensitivity of the results to the exact choice of the prior $p(\lambda)$, while keeping the advantages of the Bayesian approach.

Empirical Bayes is a two-step approach: first, the empirical Bayes choice for $\lambda$ needs to be determined; second, the model is fitted using the EB prior. In order to obtain an EB estimate for $\lambda$, we need to find the solution that maximizes the marginal likelihood ${ }^{2}$, i.e.,

$$
\lambda^{E B}=\arg \max p(\boldsymbol{y} \mid \lambda) .
$$

To obtain $\lambda^{E B}$, first note that the marginal likelihood is the product of the likelihood and prior integrated over the model parameters, i.e.,

$$
p(\boldsymbol{y} \mid \lambda)=\iiint p\left(\boldsymbol{y} \mid X, \beta_{0}, \boldsymbol{\beta}, \sigma^{2}\right) p\left(\beta_{0}\right) p\left(\boldsymbol{\beta} \mid \sigma^{2}, \lambda\right) p\left(\sigma^{2}\right) d \beta_{0} d \boldsymbol{\beta} d \sigma^{2}
$$

Instead of directly optimizing, we achieve (4) by sampling from the posterior with a noninformative prior for $\lambda^{3}{ }^{3}$ The EB estimate $\lambda^{E B}$

\footnotetext{
${ }^{2}$ The marginal likelihood quantifies the probability of observing the data given the model. Therefore, plugging in the EB estimate for $\lambda$ will result in a prior that predicts the observed data best.

${ }^{3}$ Specifically, we use $\lambda \sim$ half-Cauchy $(0,10000)$ to ensure a stable MCMC sampler.
} 


\section{BAyESian PENALIZATION}

is the mode of the marginal posterior for $\lambda$, i.e., $p(\lambda \mid \boldsymbol{y})$. This corresponds to the maximum of the marginal likelihood $p(\boldsymbol{y} \mid \lambda)$ because of the noninformative prior for $\lambda$.

3. Cross-validation. For cross-validation $(\mathrm{CV})$, the data is split into a training, validation, and test set. The goal is to find a value for $\lambda$ which results in a model that is accurate in predicting new data, i.e., a generalizable model that captures the signal in the data, but does not overfit (Hastie et al., 2015). To find this value for $\lambda$, a range of values is considered, using the training data $\boldsymbol{y}^{\text {train }}$ to fit all models with the different $\lambda$ values. Next, each resulting model is used to predict the responses in the validation set $\boldsymbol{y}^{v a l}$. The value for $\lambda$ that minimizes some loss function is selected, i.e.,

$$
\lambda^{C V}=\arg \min L\left(\boldsymbol{y}^{\text {train }}, \boldsymbol{y}^{\text {val }}\right) .
$$

Given that the loss function is the negative of the log likelihood, this is equivalent to:

$$
\lambda^{C V}=\arg \max p\left(\boldsymbol{y}^{\text {val }} \mid \boldsymbol{y}^{\text {train }}, \lambda\right) .
$$

Finally, $\lambda^{C V}$ is used to fit the model on the test set. Generally, the prediction mean squared error (PMSE) is used to determine $\lambda^{C V}$, which corresponds to a quadratic loss function.

In practice $k$-fold cross-validation is often used. $k$-fold cross-validation is a specific implementation of cross-validation in which the data is split in only a training and a test set. The training set is split in $K$ parts (usually $K=5$ or $K=10$ ) and the range of $\lambda$ values is applied $K$ times on $K-1$ parts of the training set, each time with a different part as validation set. The $K$ estimates of the PMSE are then averaged and a standard error is computed.

Frequentist penalization approaches often rely on cross-validation. In the Bayesian literature, full and empirical Bayes are often employed, although cross-validation is also possible in a Bayesian approach (see for example the loo package in R; Vehtari et al., 2018). The intuition behind empirical Bayes and cross-validation is similar: empirical Bayes aims to choose the value for $\lambda$ that is best in predicting the full data set, while cross-validation aims to choose the value for $\lambda$ that is best in predicting the validation set given a 


\section{BAYESIAN PENALIZATION}

training set. A possible disadvantage of empirical Bayes and cross-validation is that the (marginal) likelihood can be flat or multimodal when there are multiple penalty parameters (van de Wiel et al., 2017). ${ }^{4}$

Throughout this paper, we will focus on the full and empirical Bayes approach to determine $\lambda$, and only consider cross-validation for the frequentist penalization methods we will compare the priors to.

\section{Overview shrinkage priors}

In this section we will give a general overview of shrinkage priors that have been proposed in the literature. Given the extensive number of shrinkage priors that has been investigated, we will limit the overview to priors that are related to well-known classical penalization methods and shrinkage priors that are popular in the Bayesian literature. Given that most shrinkage priors fall into these categories, the resulting overview, while not exhaustive, is intended to be comprehensive and will help researchers to navigate through this literature. In total, we will discuss nine different shrinkage priors.

Many continuous, unimodal, and symmetric distributions can be parametrized as a scale mixture of normals meaning that the distribution is rewritten as a normal distribution (i.e., $\left.\operatorname{Normal}\left(\mu, \sigma^{2}\right)\right)$ where the scale parameter is given a mixing density $h\left(\sigma^{2}\right)$ (see e.g., West, 1987). Where possible, we will present the different priors in a common framework by providing the scale mixture of normals formulation for each prior. Using this formulation, the theoretical differences and similarities between the priors become more clear and, additionally, the scale mixture of normals formulation can be computationally more efficient.

We will now describe each prior in turn. The densities for several of the shrinkage priors are presented in Table 1 and plotted in Figure 1. We will consider a full and empirical Bayes approach to obtain the penalty parameter $\lambda$ (see Section 2) for all shrinkage priors, unless stated otherwise. For the full Bayesian approach, we will consider standard half-Cauchy priors for the penalty parameters as a robust default prior choice. We have included this choice for the prior on $\lambda$ in the descriptions below, but note that other choices are possible as well.

\footnotetext{
${ }^{4}$ In the initial empirical Bayes approach we used a uniform prior for $\lambda$ and this problem became evident through non-convergence of the sampler or extreme estimates for $\lambda^{E B}$. The problem was solved by using the half-Cauchy prior instead.
} 


\section{Bayesian PEnAlization}

\begin{tabular}{ccc}
\hline Shrinkage prior & Conditional prior density $p\left(\beta_{j} \mid \lambda, \ldots\right)$ & Reference \\
\hline Ridge & $p\left(\beta_{j} \mid \sigma^{2}, \lambda\right)=\sqrt{\frac{\lambda}{2 \pi \sigma^{2}}} \exp \left\{-\frac{\lambda \beta_{j}^{2}}{2 \sigma^{2}}\right\}$ & Hsiang (1975) \\
Local Student's $t$ & $p\left(\beta_{j} \mid \sigma^{2}, \lambda\right)=\frac{\sigma^{2}}{\pi \lambda}\left(1+\left(\frac{\sigma^{2}}{\lambda \beta_{j}}\right)^{2}\right)$ & Griffin and Brown (2005); \\
Lasso & $p\left(\beta_{j} \mid \sigma^{2}, \lambda\right)=\frac{\lambda}{2 \sqrt{\sigma^{2}}} \exp \left\{\frac{-\lambda\left|\beta_{j}\right|}{\sqrt{\sigma^{2}}}\right\}$ & Meuwissen et al. (2001) \\
Elastic net & $p\left(\beta_{j} \mid \sigma^{2}, \lambda_{1}, \lambda_{2}\right)=C \exp \left\{-\frac{1}{2 \sigma^{2}}\left(\lambda_{1}\left|\beta_{j}\right|+\lambda_{2} \beta_{j}^{2}\right)\right\}$ & Park and Casella (2008) \\
Group lasso & $p\left(\beta_{j} \mid \sigma, \lambda\right)=C \exp \left\{-\frac{\lambda}{\sigma} \sum_{g=1}^{G}|| \boldsymbol{\beta}_{g} \|\right\}$ & Li and Lin (2010) \\
Hyperlasso & $p\left(\beta_{j} \mid \lambda\right)=\lambda(2 \pi)^{\frac{1}{2}}\left[1-\frac{\left(\lambda\left|\beta_{j}\right|\right)\left\{(\lambda-\Phi)\left(\lambda\left|\beta_{j}\right|\right)\right\}}{\phi\left(\lambda \mid \beta_{j}\right)}\right]$ & Kyung et al. (2010) \\
Horseshoe & Not analytically tractable & Griffin and Brown (2011) \\
Discrete normal mixture & $p\left(\beta_{j} \mid \gamma_{j}, \phi_{j}^{2}\right)=\left(1-\gamma_{j}\right)\left(\frac{1}{\sqrt{2 \pi \phi_{j}^{2}}} \exp \left\{-\frac{\beta_{j}^{2}}{2 \phi_{j}^{2}}\right\}\right)+\gamma_{j}\left(\frac{1}{\pi\left(1+\beta_{j}^{2}\right)}\right)$ & Carvalho et al. (2010) \\
& Mitchell and Beauchamp (1988) \\
\hline
\end{tabular}

Note. C denotes a normalization constant. $\Phi()$ and $\phi()$ in the hyperlasso are the cumulative density function and the probability density function of the standard normal distribution.

Table 1: Conditional prior densities for the regression coefficients $\beta$ implied by the various shrinkage priors and references for each shrinkage prior.
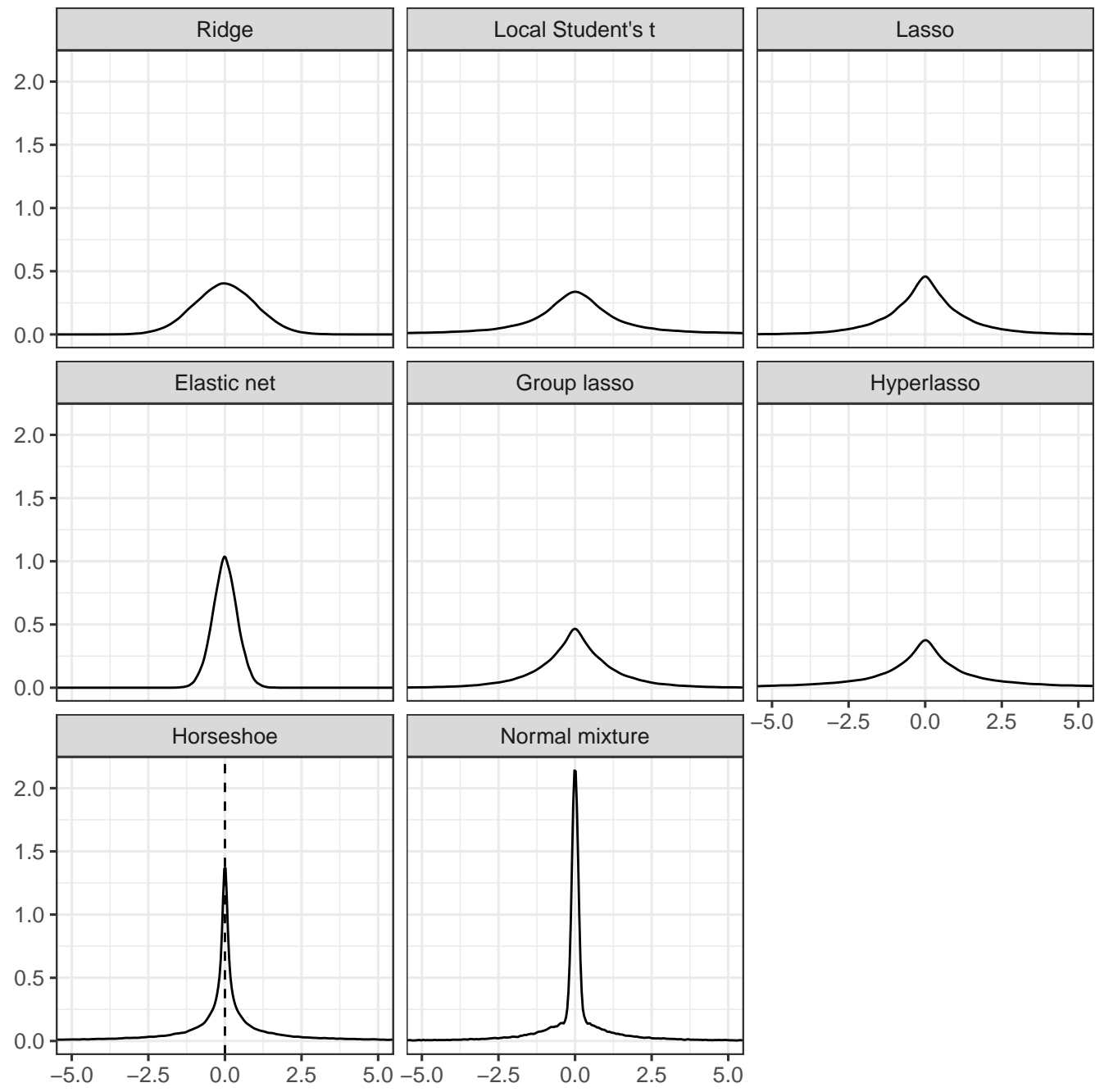

Figure 1: Densities of the shrinkage priors 


\section{BAyesian Penalization}

\subsection{Ridge}

The ridge prior corresponds to normal priors centered around 0 on the regression coefficients, i.e., (see e.g., Hsiang, 1975)

$$
\begin{aligned}
\beta_{j} \mid \lambda, \sigma^{2} & \sim \operatorname{Normal}\left(0, \frac{\sigma^{2}}{\lambda}\right), \text { for } j=1, \ldots, p . \\
\lambda & \sim \operatorname{half-Cauchy}(0,1)
\end{aligned}
$$

The posterior mean estimates under this prior will correspond to estimates obtained using the ridge penalty or $l_{2}$ norm, i.e., $q=2$ in Equation (1) (Hoerl and Kennard, 1970). The penalty parameter $\lambda$ determines the amount of shrinkage, with larger values resulting in smaller prior variation and thus more shrinkage of the coefficients towards zero.

\subsection{Local Student's $t$}

We can extend the ridge prior in Equation 8 by making the prior variances predictor-specific, thereby allowing for more variation, i.e.,

$$
\begin{aligned}
\beta_{j} \mid \tau_{j}^{2} & \sim \operatorname{Normal}\left(0, \sigma^{2} \tau_{j}^{2}\right) \\
\tau_{j}^{2} \mid \nu, \lambda & \sim \operatorname{Inverse-Gamma}\left(\frac{\nu}{2}, \frac{\nu}{2 \lambda}\right), \text { for } j=1, \ldots, p, \\
\lambda & \sim \operatorname{half-Cauchy}(0,1)
\end{aligned}
$$

When integrating $\tau_{j}^{2}$ out, the following conditional prior distribution for the regression coefficients is obtained:

$$
\beta_{j} \mid \nu, \lambda, \sigma^{2} \sim \operatorname{Student}\left(\nu, 0, \frac{\sigma^{2}}{\lambda}\right)
$$

where Student $\left(\nu, 0, \frac{\sigma^{2}}{\lambda}\right)$ denotes a non-standardized Student's $t$ distribution centered around 0 with $\nu$ degrees of freedom and scale parameter $\frac{\sigma^{2}}{\lambda}$. A smaller value for $\nu$ results in a distribution with heavier tails, with $\nu=1$ implying a Cauchy prior for $\beta_{j}$. Larger (smaller) values for $\lambda$ result in more (less) shrinkage towards $m$. This prior has been considered, among others, by Griffin and Brown (2005) and Meuwissen et al. (2001). Compared to the ridge prior in (8), the local Student's $t$ prior has heavier tails. Throughout this paper, we will consider $\nu=1$, such that the prior has Cauchy-like tails. 


\section{BAyesian Penalization}

\subsection{Lasso}

The Bayesian counterpart of the lasso penalty was first proposed by Park and Casella (2008). The Bayesian lasso can be obtained as a scale mixture of normals with an exponential mixing density, i.e.,

$$
\begin{aligned}
& \beta_{j} \mid \tau_{j}^{2}, \sigma^{2} \sim \operatorname{Normal}\left(0, \sigma^{2} \tau_{j}^{2}\right) \\
& \tau_{j}^{2} \mid \lambda^{2} \sim \operatorname{Exponential}\left(\frac{\lambda^{2}}{2}\right), \text { for } j=1, \ldots, p, \\
& \lambda \sim \operatorname{half-Cauchy}(0,1)
\end{aligned}
$$

Integrating $\tau_{j}^{2}$ out results in double-exponential or Laplace priors on the regression coefficients, i.e.,

$$
\beta_{j} \mid \lambda, \sigma \sim \text { Double-exponential }\left(0, \frac{\sigma}{\lambda}\right) \text {, for } j=1, \ldots, p \text {. }
$$

With this prior, the posterior mode estimates are similar to estimates obtained under the lasso penalty or $l_{1}$ norm, i.e., $q=1$ in Equation (1) (Tibshirani, 1996). In addition to the overall shrinkage parameter $\lambda$, the lasso prior has an additional predictor-specific shrinkage parameter $\tau_{j}$. Therefore, the lasso prior is more flexible than the ridge prior which only relies on the overall shrinkage parameter in (8). Figure 1 clearly shows that the lasso prior has a sharper peak around zero compared to the ridge prior.

\section{Disadvantages of the lasso}

The popularity of the classical lasso lies in its ability to shrink coefficients to zero, thereby automatically performing variable selection. However, there are several disadvantages to the classical lasso. Specifically, (i) it cannot select more predictors than observations, which is problematic when $p>n$; (ii) when a group of predictors is correlated, the lasso generally selects only one predictor of that group; (iii) the prediction error is higher for the lasso compared to the ridge when $n>p$ and the predictors are highly correlated; (iv) it can lead to overshrinkage of large coefficients (see e.g., Polson and Scott, 2011); and (v) it does not always have the oracle property, which implies it does not always perform as well in terms of variable selection as if the true underlying model has been given (Fan and Li, 2001). The lasso only enjoys the oracle property under specific and stringent conditions (Fan and Li, 2001; Zou, 2006). These disadvantages have sparked the development of several generalizations of the lasso. We will now discuss the Bayesian counterparts 


\section{BAYESIAN PENALIZATION}

of several of these generalizations, including the elastic net, group lasso, and hyperlasso. Note that for the Bayesian lasso, coefficients cannot become exactly zero and thus a criterion is needed to select the relevant variables. Depending on the criterion used, more predictors than observations could be selected. However, the Bayesian lasso does not allow a grouping structure to be included, it overshrinks large coefficients, and it does not have the oracle property since the tails for the prior on $\beta_{j}$ are not heavier than exponential tails (Polson et al., 2014).

\subsection{Elastic net}

The most popular generalization of the lasso is the elastic net (Zou and Hastie, 2005). The elastic net can be seen as a combination of the ridge and lasso. The elastic net resolves issues (i), (ii), and (iii) of the ordinary lasso. The elastic net prior can be obtained as the following scale mixture of normals (Li and Lin, 2010):

$$
\begin{aligned}
\beta_{j} \mid \lambda_{2}, \tau_{j}, \sigma^{2} & \sim \operatorname{Normal}\left(0,\left(\frac{\lambda_{2}}{\sigma^{2}} \frac{\tau_{j}}{\tau_{j}-1}\right)^{-1}\right) \\
\tau_{j} \mid \lambda_{2}, \lambda_{1}, \sigma^{2} & \sim \text { Truncated-Gamma }\left(\frac{1}{2}, \frac{8 \lambda_{2} \sigma^{2}}{\lambda_{1}^{2}}\right), \text { for } j=1, \ldots, p, \\
\lambda_{1} & \sim \text { half-Cauchy }(0,1) \\
\lambda_{2} & \sim \text { half-Cauchy }(0,1)
\end{aligned}
$$

where the truncated Gamma density has support $(1, \infty)$. This implies the following conditional prior distributions for the regression coefficients:

$$
\begin{gathered}
p\left(\beta_{j} \mid \sigma^{2}, \lambda_{1}, \lambda_{2}\right)=C\left(\lambda_{1}, \lambda_{2}, \sigma^{2}\right) \exp \left\{-\frac{1}{2 \sigma^{2}}\left(\lambda_{1}\left|\beta_{j}\right|+\lambda_{2} \beta_{j}^{2}\right)\right\}, \\
\text { for } j=1, \ldots, p,
\end{gathered}
$$

where $C\left(\lambda_{1}, \lambda_{2}, \sigma^{2}\right)$ denotes the normalizing constant. The corresponding posterior modes for $\beta_{j}$ are equivalent to the estimates from the classical elastic net penalty. Expression (14) illustrates how the elastic net prior offers a combination of the double-exponential prior, i.e., the lasso penalty $\lambda\left|\beta_{j}\right|$, and the normal prior, i.e., the ridge penalty $\lambda \beta_{j}^{2}$. Specifically, the two penalty parameters $\lambda_{1}$ and $\lambda_{2}$ determine the relative influence of the lasso and ridge penalty, respectively. This can also be seen in Figure 1: the elastic net is not as sharply peaked as the lasso prior, but it is sharper than the ridge prior. 


\section{BAYESIAN PENALIZATION}

As mentioned in the Introduction, a disadvantage of the classical elastic net is that the sequential cross-validation procedure used to determine the penalty parameters results in overshrinkage of the coefficients. This problem is resolved in the Bayesian approach by estimating both penalty parameters simultaneously through a full or empirical Bayes approach.

\subsection{Group lasso}

The group lasso (Yuan and Lin, 2006) is a generalization of the lasso primarily aimed at improving performance when predictors are grouped in some way, for example when qualitative predictors are coded as dummy or one-hot variables (as is often implicitly done in ANOVA, for instance). Similarly to the elastic net, the penalty function induced by the group lasso lies between the $l_{1}$ penalty of the lasso in (12) and the $l_{2}$ penalty of the ridge in (8). To apply the group lasso, the vector of regression coefficients $\beta$ is split in $G$ vectors $\beta_{g}$, where each vector represents the coefficients of predictors in that group. Denote by $m_{g}$ the dimension of each vector $\boldsymbol{\beta}_{g}$. The group lasso corresponds to the following scale mixture of normals (Kyung et al., 2010):

$$
\begin{aligned}
\boldsymbol{\beta}_{g} \mid \tau_{g}^{2}, \sigma^{2} & \sim \operatorname{MVN}\left(\mathbf{0}, \sigma^{2} \tau_{g}^{2} I_{m_{g}}\right) \\
\tau_{g}^{2} \mid \lambda^{2} & \sim \operatorname{Gamma}\left(\frac{m_{g}+1}{2}, \frac{\lambda^{2}}{2}\right), \text { for } g=1, \ldots, G, \\
\lambda & \sim \operatorname{half-Cauchy}(0,1)
\end{aligned}
$$

where MVN denotes the multivariate normal distribution with dimension $m_{g}$ and $I_{m_{g}}$ denotes an $\left(m_{g} \times m_{g}\right)$ identity matrix. Note that, contrary to the priors considered thus far, the group lasso prior does not consist of independent priors on the regression coefficients $\beta_{j}$, but rather independent priors on the groups of regression coefficients $\boldsymbol{\beta}_{g}$. If there is no grouping structure, $m_{g}=1$ and the Bayesian group lasso in (15) reduces to the Bayesian lasso in (11). The scale mixture of normals in (15) leads to the following conditional prior for the regression coefficients (Kyung et al., 2010):

$$
p\left(\beta_{j} \mid \sigma^{2}, \lambda\right)=C \exp \left\{-\frac{\lambda}{\sqrt{\sigma^{2}}} \sum_{g=1}^{G}\left\|\boldsymbol{\beta}_{g}\right\|\right\} \text {, for } g=1, \ldots, G \text {, and } j=1, \ldots, p \text {, }
$$

where $\left\|\beta_{g}\right\|=\left(\beta_{g}^{\prime} \beta_{g}\right)^{\frac{1}{2}}$ and $C$ denotes the normalizing constant. Due to the simultaneous penalization of all coefficients in one group, all estimated 


\section{BAYESIAN PENALIZATION}

regression coefficients in one group will be either zero or nonzero, depending on the value for $\lambda$.

\subsection{Hyperlasso}

Zou (2006) proposes the adaptive lasso as a generalization of the lasso that enjoys the oracle property (limitation (v) of the lasso), i.e., it performs as well as if the true underlying model has been given. The central idea of the adaptive lasso is to separately weigh the penalty for each coefficient based on the observed data. A Bayesian adaptive lasso has been proposed, among others, by Alhamzawi et al. (2012) and Feng et al. (2015). However, as noted by Griffin and Brown (2011), the weights included in the adaptive lasso place great demands on the data, which can lead to poor performance in terms of prediction and variable selection when the sample size is small. Therefore, Griffin and Brown (2011) propose the hyperlasso as a Bayesian alternative to the adaptive lasso, which is obtained through the following mixture of normals:

$$
\begin{aligned}
\beta_{j} \mid \phi_{j}^{2} & \sim \operatorname{Normal}\left(0, \phi_{j}^{2}\right) \\
\phi_{j}^{2} \mid \tau_{j} & \sim \operatorname{Exponential}\left(\tau_{j}\right) \\
\tau_{j} \mid \nu, \lambda^{2} & \sim \operatorname{Gamma}\left(\nu, \frac{1}{\lambda^{2}}\right) \text { for } j=1, \ldots, p . \\
\lambda & \sim \operatorname{half-Cauchy}(0,1)
\end{aligned}
$$

This is equivalent to placing a Gamma mixing density on the hyperparameter of the double-exponential prior:

$$
\begin{aligned}
\beta_{j} \mid \tau_{j} & \sim \text { Double-Exponential }\left(0,\left(2 \tau_{j}\right)^{1 / 2}\right) \\
\tau_{j} \mid \nu, \lambda^{2} & \sim \operatorname{Gamma}\left(\nu, \frac{1}{\lambda^{2}}\right), \text { for } j=1, \ldots, p .
\end{aligned}
$$

Note that the density of the hyperlasso prior strongly resembles the density of the lasso prior (Figure 1), the main difference being that the hyperlasso has heavier tails than the lasso. Contrary to the priors considered thus far, this prior corresponds to a penalty that is non-convex implying that multiple posterior modes can exist. Therefore, care must be taken to ensure that the complete posterior distribution is explored. In addition, the hyperlasso prior for $\beta$ is not conditioned on the error variance $\sigma^{2}$. Following Griffin and Brown (2011), we will consider the specific case of $\nu=0.5$. However, whereas Griffin and Brown (2011) use cross-validation to choose $\lambda$, we will rely on a full and empirical Bayes approach. 


\section{BAyesian Penalization}

\subsection{Horseshoe}

A popular shrinkage prior in the Bayesian literature is the horseshoe prior (Carvalho et al., 2010):

$$
\begin{aligned}
\beta_{j} \mid \tau_{j}^{2} & \sim \operatorname{Normal}\left(0, \tau_{j}^{2}\right) \\
\tau_{j} \mid \lambda & \sim \operatorname{Half-Cauchy}(0, \lambda), \text { for } j=1, \ldots, p \\
\lambda \mid \sigma & \sim \operatorname{Half-Cauchy}(0, \sigma) .
\end{aligned}
$$

Note that Carvalho et al. (2010) explicitly include the half-Cauchy prior for $\lambda$ in their specification, thereby implying a full Bayes approach. This formulation results in a horseshoe prior that is automatically scaled by the error standard deviation $\sigma$. The half-Cauchy prior can be written as a mixture of inverse Gamma and Gamma densities, so that the horseshoe prior in (19) can be equivalently specified as:

$$
\begin{aligned}
\beta_{j} \mid \tau_{j}^{2} & \sim \operatorname{Normal}\left(0, \tau_{j}^{2}\right) \\
\tau_{j}^{2} \mid \omega & \sim \operatorname{inverse} \operatorname{Gamma}\left(\frac{1}{2}, \omega\right) \\
\omega \mid \lambda^{2} & \sim \operatorname{Gamma}\left(\frac{1}{2}, \lambda^{2}\right) \\
\lambda^{2} \mid \gamma & \sim \operatorname{inverse} \operatorname{Gamma}\left(\frac{1}{2}, \gamma\right) \\
\gamma \mid \sigma^{2} & \sim \operatorname{Gamma}\left(\frac{1}{2}, \sigma^{2}\right)
\end{aligned}
$$

An expression for the marginal prior of the regression coefficients $\beta_{j}$ is not analytically tractable. The name "horseshoe" prior arises from the fact that for fixed values $\lambda=\sigma=1$, the implied prior for the shrinkage coefficient $\kappa_{j}=$ $\frac{1}{1+\tau_{j}^{2}}$ is similar to a horseshoe shaped $\operatorname{Beta}(0.5,0.5)$ prior. Large coefficients will lead to a shrinkage coefficient $\kappa_{j}$ that is close to zero such that there is practically no shrinkage, whereas small coefficients will have a $\kappa_{j}$ close to 1 and will be shrunken heavily. Note that the horseshoe prior is the only prior with an asymptote at zero (Figure 1). Combined with the heavy tails, this ensures that small coefficients are heavily shrunken towards zero while large coefficients remain large. The horseshoe prior has also been termed a global-local shrinkage prior (e.g., Polson and Scott, 2011) because it has a predictor-specific local shrinkage component $\tau_{j}$ as well as a global shrinkage component $\lambda$. The basic intuition is that the global shrinkage parameter $\lambda$ performs shrinkage on all coefficients and the local shrinkage parameters $\tau_{j}$ 


\section{BAYESIAN PENALIZATION}

loosen the amount of shrinkage for truly large coefficients. Many global-local shrinkage priors (including the horseshoe and hyperlasso) are special cases of the general class of hypergeometric inverted-beta distributions (Polson and Scott, 2012). In addition to the full Bayes approach implied by the specification in (19), we will also consider an empirical Bayes approach to determine $\lambda$.

\subsection{Regularized horseshoe}

The horseshoe prior in Subsection 3.7 has the characteristic that large coefficients will not be shrunken towards zero too heavily. Indeed, this is one of the advertised qualities of the horseshoe prior (Carvalho et al., 2010). Although this property is desirable in theory, it can be problematic in practice, especially when parameters are weakly identified. In this situation, the posterior means of the regression coefficients might not exist and even if they do, the horseshoe prior can result in an unstable MCMC sampler (Ghosh et al., 2017). To solve these problems Piironen and Vehtari (2017) propose the regularized horseshoe, which is defined as follows:

$$
\begin{aligned}
& \beta_{j} \mid \tilde{\tau}_{j}^{2}, \lambda \sim \operatorname{Normal}\left(0, \tilde{\tau}_{j}^{2} \lambda\right), \text { with } \tilde{\tau}_{j}^{2}=\frac{c^{2} \tau_{j}^{2}}{c^{2}+\lambda^{2} \tau_{j}^{2}} \\
& \lambda \mid \lambda_{0}^{2} \sim \text { half-Cauchy }\left(0, \lambda_{0}^{2}\right), \text { with } \lambda_{0}=\frac{p_{0}}{p-p_{0}} \frac{\sigma}{\sqrt{n}} \\
& \tau_{j} \sim \operatorname{half-Cauchy}(0,1) \\
& c^{2} \mid \nu, s^{2} \sim \text { inverse } \operatorname{Gamma}\left(\nu / 2, \nu s^{2} / 2\right),
\end{aligned}
$$

where $p_{0}$ represents a prior guess of the number of relevant variables. The resulting prior will shrink small coefficients in the same way as the horseshoe ${ }^{5}$, but unlike the horseshoe, large coefficients will be shrunken towards zero by a Student's $t$ distribution with $\nu$ degrees of freedom and scale $s^{2}$. Piironen and Vehtari (2017) use a Student's $t$ distribution with $\nu=4$ and $s^{2}=2$ and we use the same hyperparameters, although other choices are possible. As long as the degrees of freedom $\nu$ are small enough, the tails will be heavy enough to ensure a robust shrinkage pattern for large coefficients.

It is possible to specify a half-Cauchy prior for the global shrinkage parameter with a scale equal to 1 or the error standard deviation, i.e., $\lambda \sim$ half-Cauchy $(0,1)$ or $\lambda \sim$ half-Cauchy $(0, \sigma)$, for example when no prior

\footnotetext{
${ }^{5}$ Because of the similarity to the horseshoe, the density and contour plots for the regularized horseshoe are not substantially different from those of the horseshoe and are therefore not included in Figure 1 and Figure 3.
} 


\section{BAYESIAN PENALIZATION}

information is available regarding the number of relevant variables. However, as noted by Piironen and Vehtari (2017), the scale based on the a priori number of relevant variables will generally be much smaller than 1 or $\sigma$. In addition, even if the prior guess for the number of relevant parameters $p_{0}$ is incorrect, the results are robust to this choice as long as a half-Cauchy prior is used. Following the recommendations of Piironen and Vehtari (2017), we will only consider a full Bayes approach to determine $\lambda$ in the regularized horseshoe.

\subsection{Discrete normal mixture}

The normal mixture prior is a discrete mixture of a peaked prior around zero (the spike) and a vague proper prior (the slab); it is therefore also termed a spike-and-slab prior. It is substantially different from the priors considered thus far, which are all continuous mixtures of normal densities. Based on the data, regression coefficients close to zero will be assigned to the spike, resulting in shrinkage towards 0 , while coefficients that deviate substantially from zero will be assigned to the slab, resulting in (almost) no shrinkage. Early proposals of mixture priors can be found in George and McCulloch (1993) and Mitchell and Beauchamp (1988), and a scale mixture of normals formulation can be found in Ishwaran and Rao (2005). We will consider the following specification of the mixture prior:

$$
\begin{aligned}
\beta_{j} \mid \gamma_{j}, \tau_{j}^{2}, \phi_{j}^{2} & \sim\left(\gamma_{j}\right) \operatorname{Normal}\left(0, \tau_{j}^{2}\right)+\left(1-\gamma_{j}\right) \operatorname{Normal}\left(0, \phi_{j}^{2}\right) \\
\tau_{j}^{2} & \sim \text { inverse } \operatorname{Gamma}(0.5,0.5), \text { for } j=1, \ldots, p,
\end{aligned}
$$

where $\tau_{j}$ is given a vague prior so that the variance of the slab is estimated based on the data and $\phi_{j}^{2}$ is fixed to a small number, say $\phi_{j}^{2}=0.001$, to create the spike. By assigning an inverse $\operatorname{Gamma}(0.5,0.5)$ prior on $\tau_{j}^{2}$, the resulting marginal distribution of the slab component of the mixture is a Cauchy distribution.

There are several options for the prior on the mixing parameter $\gamma_{j}$. In this paper, we will consider the following two options: 1) $\gamma_{j}$ as a Bernoulli distributed variable taking on the value 0 or 1 with probability 0.5 , i.e., $\gamma_{j} \sim \operatorname{Bernoulli}(0.5)$; and 2) $\gamma_{j}$ uniformly distributed between 0 and 1, i.e., $\gamma_{j} \sim \operatorname{Uniform}(0,1)$. In the first option, which we label the Bernoulli mixture, each coefficient $\beta_{j}$ is given either the slab or the spike as prior. The second option, labelled the uniform mixture, is more flexible in that each coefficient is given a prior consisting of a mixture of the spike and slab, with each component weighted by the uniform probabilities $\gamma_{j}$ 
The density of the normal mixture prior is presented in Figure 1, which clearly shows the prior is a combination of two densities. The representation in Figure 1 is based on a normal mixture with equal mixing probabilities, rather than a Bernoulli or uniform prior on the mixing probabilities. Note that the mixture prior is not conditioned on the error variance $\sigma^{2}$. We will only consider a full Bayesian approach for the mixture priors.

\section{Illustrating the behavior of the shrinkage priors}

\subsection{Contour plots}

Contour plots provide an insightful way to illustrate the behavior of classical penalties and Bayesian shrinkage priors. First, consider Figure 2 which shows the frequentist and Bayesian contour plots for the lasso. In both plots, the green elliptical lines represent the contours of the sum of squared residuals, centered around the regular OLS estimate $\hat{\beta}_{O L S}$. The solid black diamond in the left plot represents the constraint region for the classical lasso penalty function for two predictors $\beta_{1}$ and $\beta_{2}$. The classical penalized regression

solution $\hat{\beta}_{L A S S O}$ is the point where the contour of the sum of squared residuals meets the constraint region. This point corresponds to the minimum of the penalized regression equation in (1). In the right plot, the diamond shaped contours reflect the shape of the lasso prior (Section 3.3). The contour of the Bayesian posterior distribution based on the lasso prior is shown in blue. As can be seen, the posterior distribution is located between the sum of squared residuals contour and the prior contour. The Bayesian posterior median estimate $\hat{\beta}_{B A Y E S}$ is added in blue and shrunken towards zero compared to the OLS estimate $\hat{\beta}_{O L S}$. Note that the posterior mode would correspond to the classical penalized regression solution, if the same value for the penalty parameter $\lambda$ is used. 


\section{BAyesian Penalization}
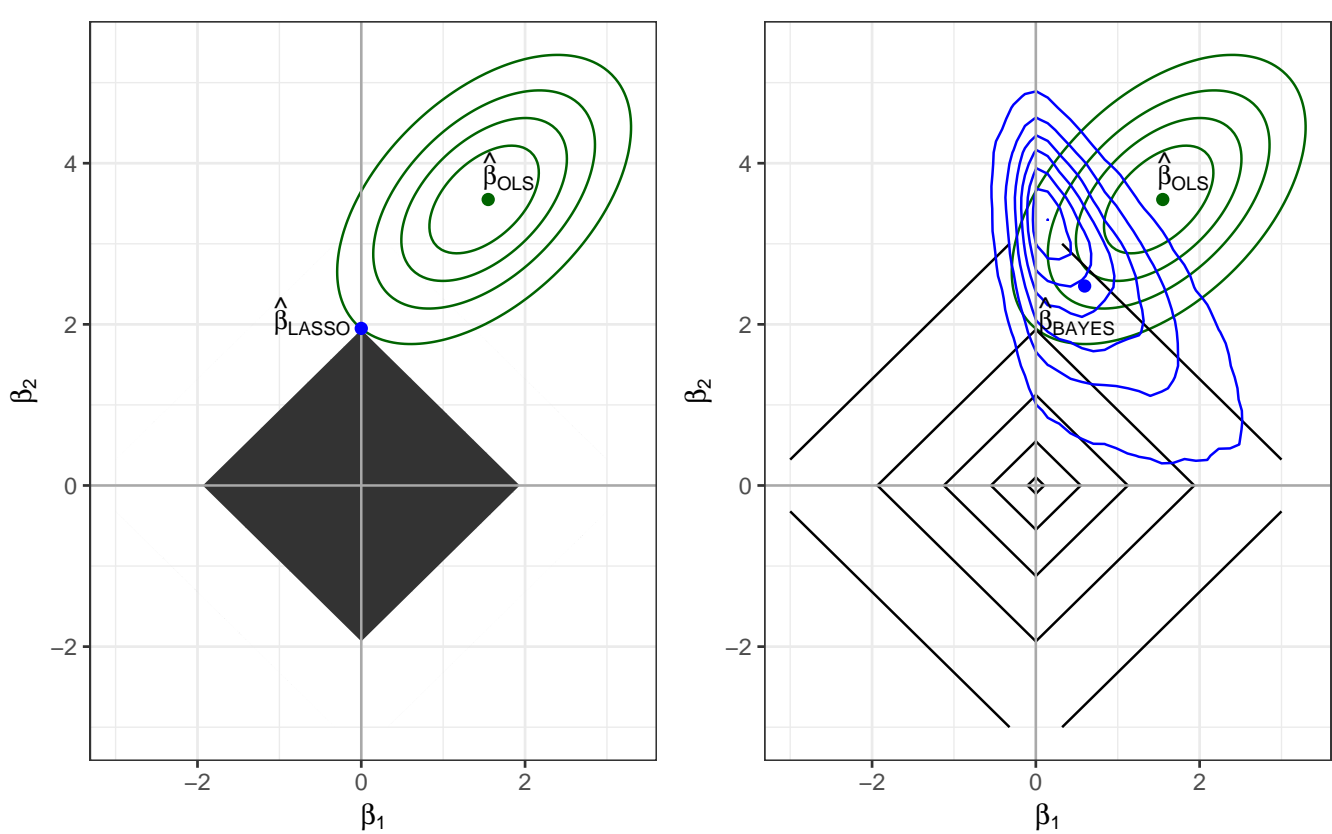

Figure 2: Contour plot representing the sum of squared residuals, classical lasso constraint region (left), bivariate lasso prior and posterior distribution (right), and the classical and Bayesian penalized point estimates.

Figure 3 shows the contour plots of the different shrinkage priors for two predictors $\beta_{1}$ and $\beta_{2}$, while Figure 4 shows the contour plots for the lasso and group lasso for three predictors. From a classical penalization perspective, the lasso and elastic net penalties have sharp corners at $\beta_{1}=\beta_{2}=0$. As a result, the contour of the sum of squared residuals will meet the contours of these penalties more easily at a point where one of the coefficients equals zero, which explains why these penalties can shrink coefficients to exactly zero. The ridge penalty, on the other hand, does not show these sharp corners and can therefore not shrink coefficients to exactly zero. From a Bayesian penalization perspective, the bivariate prior contour plots illustrate the shrinkage behavior of the priors. For example, the hyperlasso and horseshoe have a lot of prior mass where at least one element is close to zero, while the ridge has most prior mass where both elements are close to zero. Figure 3 also shows that the ridge, local Student's $t$, lasso, and elastic net are convex. This can be seen when drawing a straight line from one point to another point on a contour. For a convex distribution, the line lies completely within the contour. The hyperlasso and horseshoe prior are nonconvex, which can be seen from the starlike shape of the contour. Frequentist penalization has generally focused on convex penalties, due to their computa- 
tional convenience for optimization procedures. In the Bayesian framework, which relies on sampling (MCMC) techniques, the use of convex and nonconvex priors is computationally similar. It is recommendable, however, to use multiple starting values in the case of non-convex priors due to possible multimodality of the posterior distribution (Griffin and Brown, 2011).

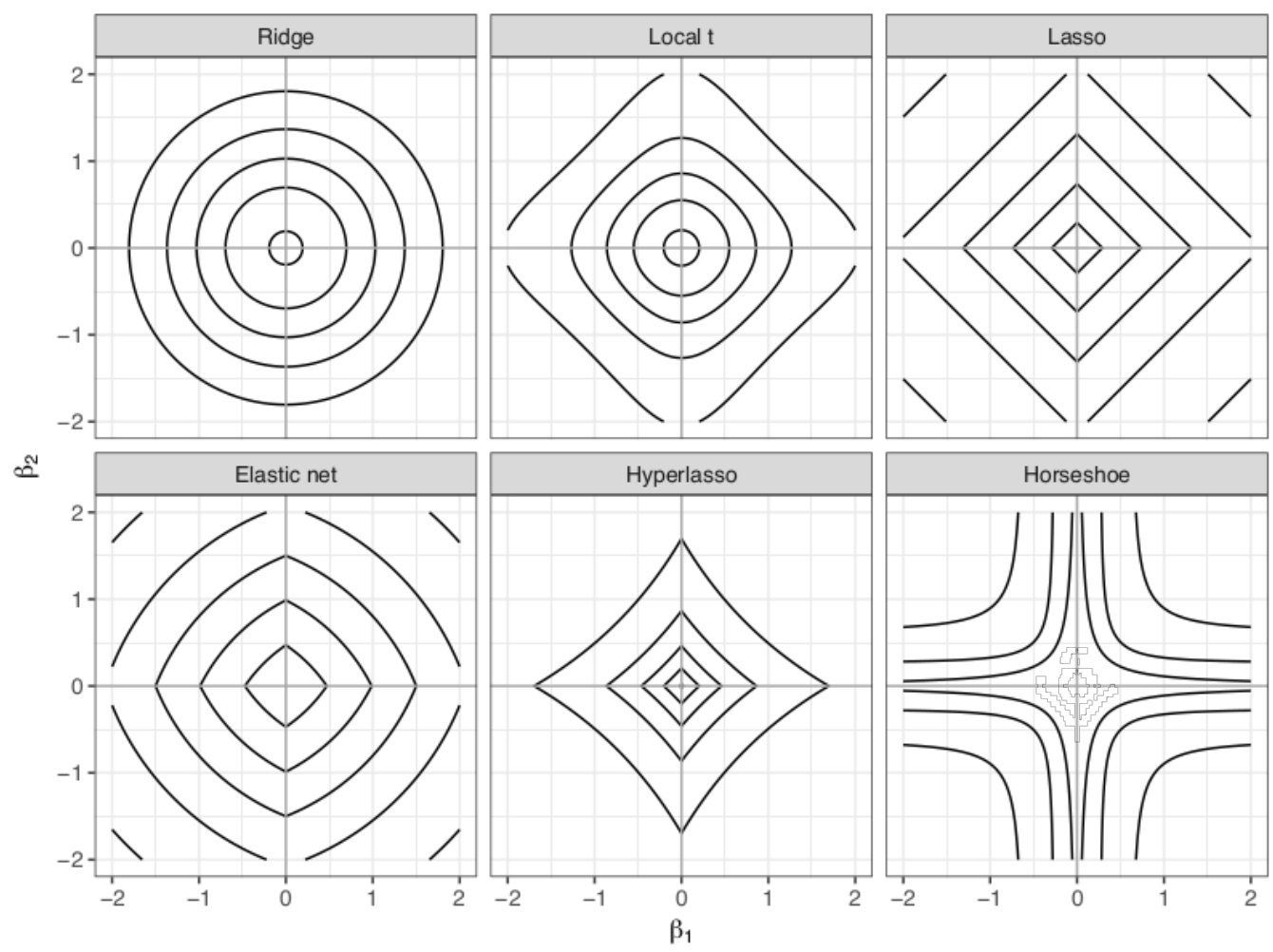

Figure 3: Contour plots representing the bivariate prior distribution of the shrinkage priors 

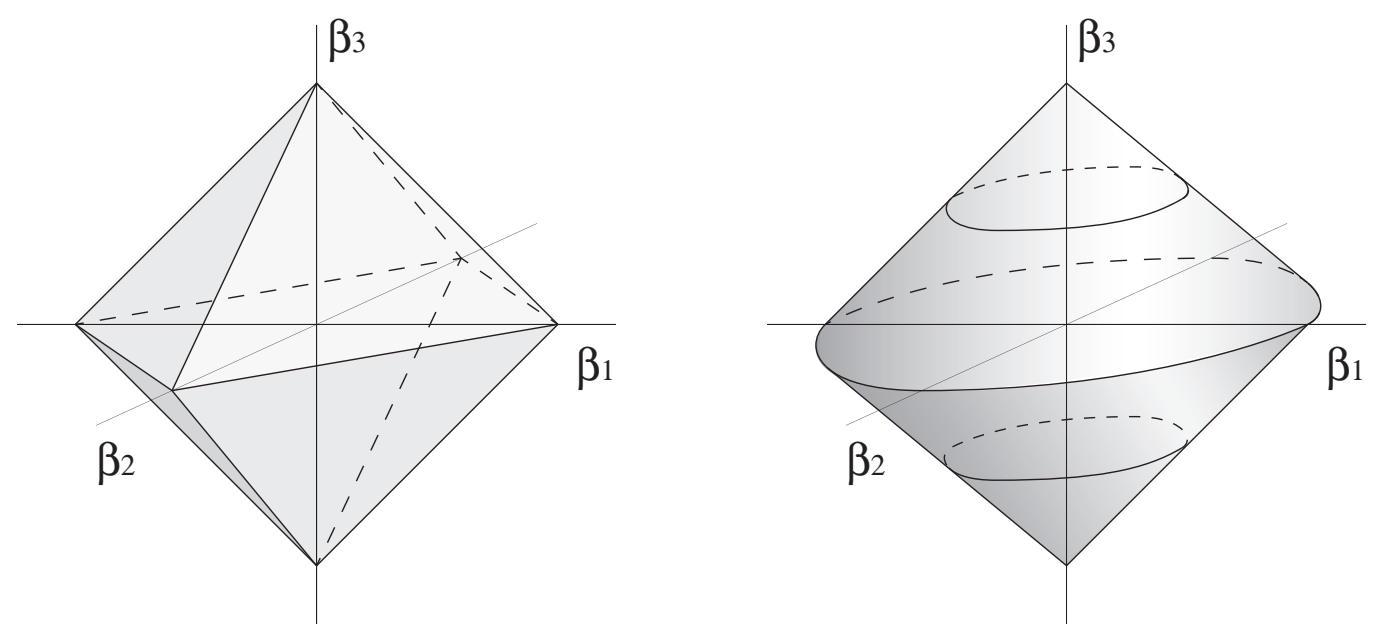

Figure 4: Contour plots of the lasso (left) and group lasso (right) in $\mathbf{R}^{3}$, with $\beta_{1}$ and $\beta_{2}$ belonging to group 1 and $\beta_{3}$ belonging to group 2. For the group lasso, if we consider only $\beta_{1}$ and $\beta_{2}$, which belong to the same group, the contour resembles that of the ridge with most prior mass if both $\beta_{1}$ and $\beta_{2}$ are close to zero. On the other hand, if we consider $\beta_{1}$ and $\beta_{3}$, which belong to different groups, the contour is similar to that of the lasso, which has more prior mass where only one element is close to zero. This illustrates how the group lasso simultaneously shrinks elements belonging to the same group. 


\subsection{Shrinkage behavior}

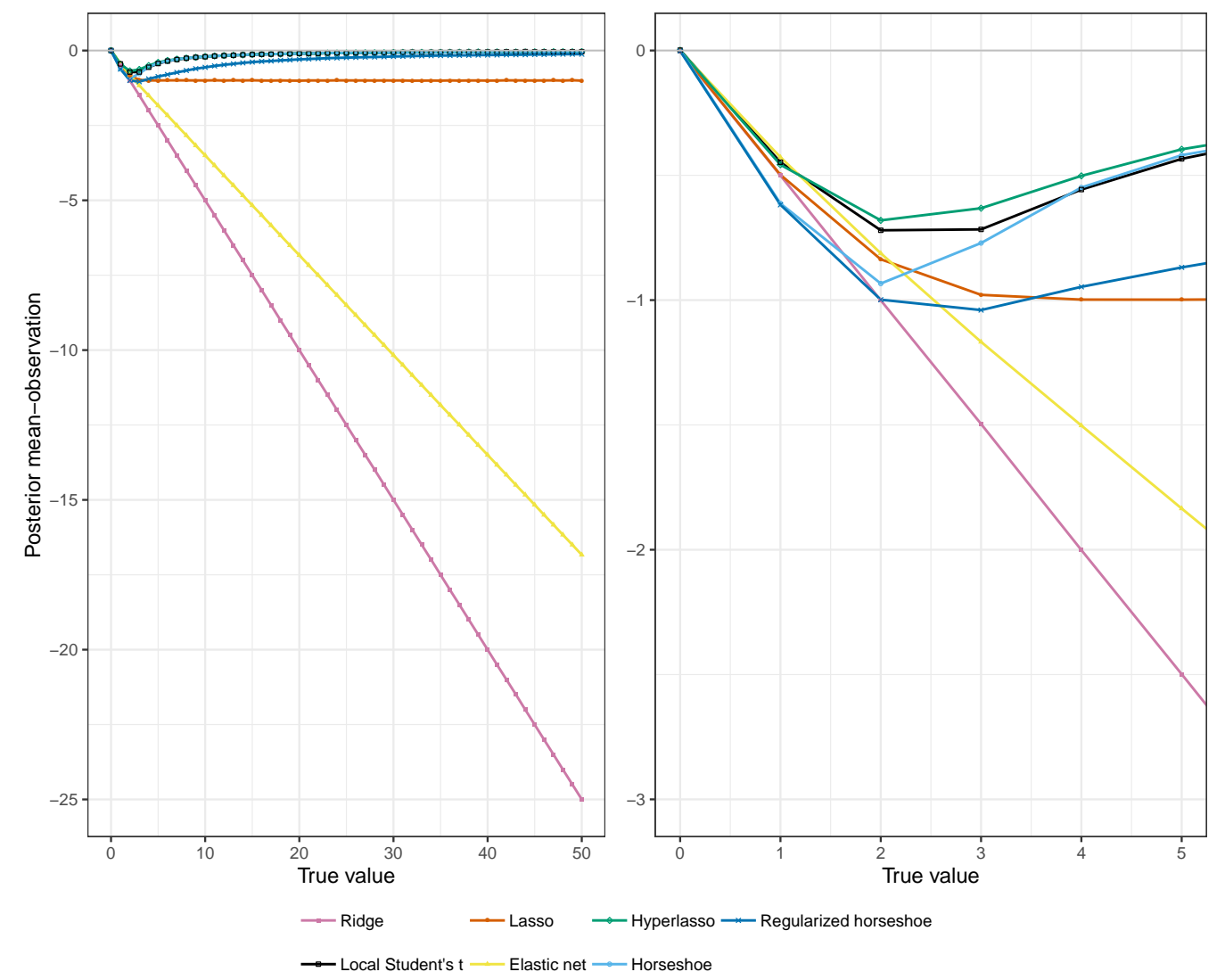

Figure 5: Difference between the estimated and true effect for the shrinkage priors in a simple normal model with the penalty parameter $\lambda$ fixed to 1 .

Prior shrinkage of small effects towards zero is important to obtain sparse solutions. Figure 5 illustrates the shrinkage behavior of the priors in a simple normal model: $y \sim \operatorname{Normal}(\beta, 1)$. We estimate $\beta$ based on a single observation $y$, which is varied from 0 to 50 . Using only a single observation is possible because the variance is known. The penalty parameter $\lambda$ for each shrinkage prior is fixed to 1 . The resulting difference between the posterior mean estimates and true means is shown in Figure 5. The behavior of the priors varies greatly. Specifically, for the ridge and elastic net priors, the difference between the estimated and true effect increases as the true mean increases. For the lasso prior, the difference increases for small effects and then remains constant. Note how the difference for the elastic net lies between the difference obtained under the ridge and lasso priors, illustrating that the elastic net is a combination of the ridge and lasso priors. The other 
shrinkage priors all show some differences between estimated and true means for small effects, indicating shrinkage of these effects towards zero, but the difference is practically zero for large effects. The right column of Figure 5 provides the same figure, but zoomed in on the small effects. Note how the regularized horseshoe shrinks large effects more than the horseshoe prior, but goes to zero eventually.

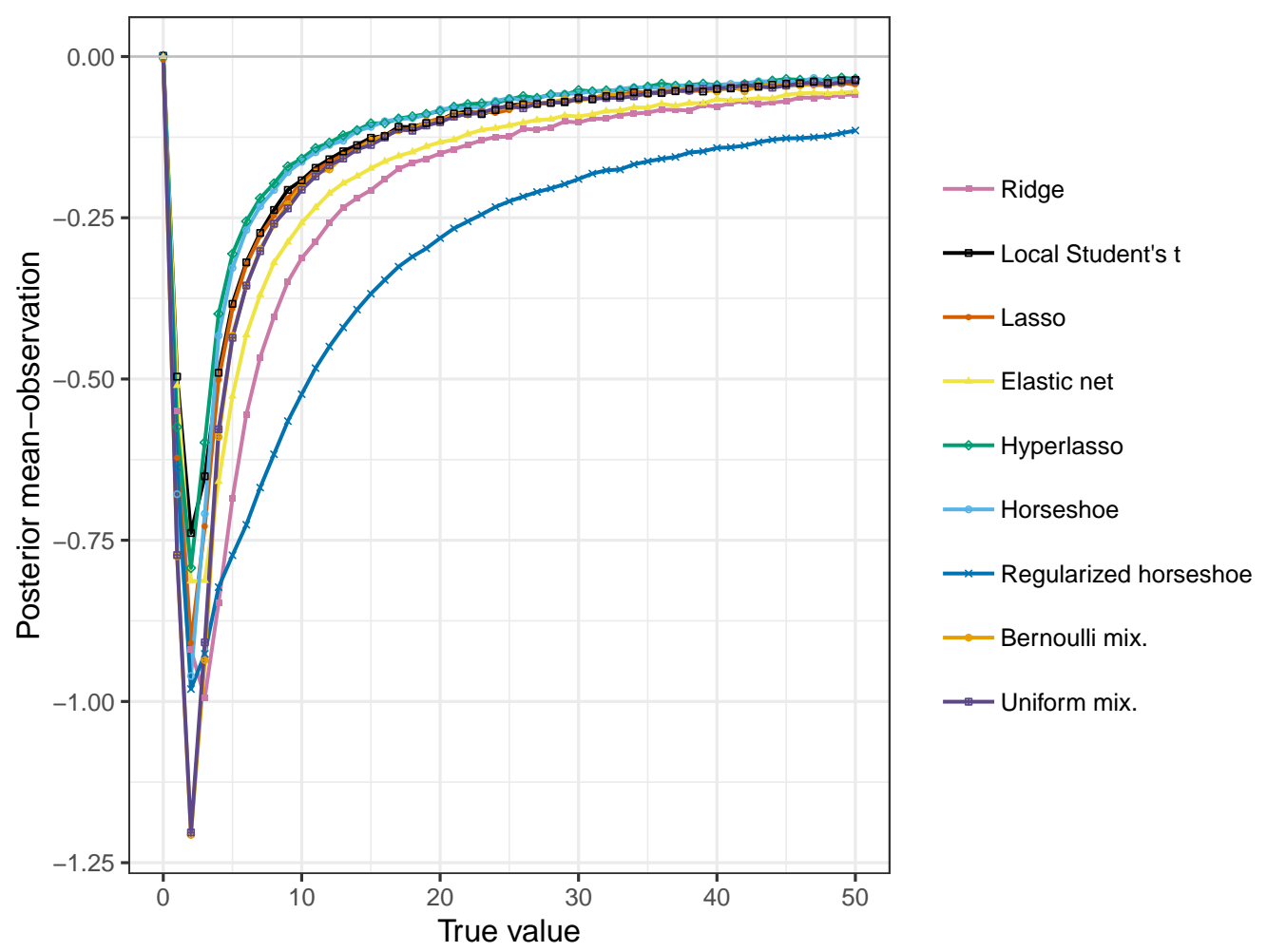

Figure 6: Difference between the estimated and true effect for the shrinkage priors in a simple normal model with a half-Cauchy hyperprior specified for the penalty parameter $\lambda$.

A similar illustration is presented in Figure 6, but based on a full Bayes approach where $\lambda$ is freely estimated. Thus, instead of fixing $\lambda$ to a specific value, it is given a standard half-Cauchy prior distribution and estimated simultaneously with the other parameters in the model. Overall, all shrinkage priors show differences between true and estimated means for small effects, which decrease towards zero as the effect grows. Note that the difference is negative, indicating that the estimated mean is smaller than the true mean. Thus, all shrinkage priors heavily pull small effects towards zero, while asserting almost no influence on larger effects, although some shrinkage still 


\section{BAYESIAN PENALIZATION}

occurs even when the true mean equals 50. The mixture priors result in the largest differences between true and estimated small effects, indicating the most shrinkage, and the local Student's $t$ prior shows the smallest difference for small effects. As the effect grows, the regularized horseshoe prior results in estimates farthest from the true effects, indicating the most shrinkage for large effects.

These illustrations indicate that when the penalty parameter is fixed, only the local Student's $t$, hyperlasso, and (regularized) horseshoe priors allow for shrinkage of small effects while estimating large effects correctly. However, if a prior is specified for the penalty parameter, so that the uncertainty in this parameter is taken into account, all shrinkage priors show this desirable behavior.

\section{Simulation study}

\subsection{Conditions}

We conduct a Monte Carlo simulation study to compare the performance of the shrinkage priors and several frequentist penalization methods. We simulate data from the linear regression model, given by: $\boldsymbol{y}=\beta_{0} \mathbf{1}+\boldsymbol{X} \boldsymbol{\beta}+\boldsymbol{\epsilon}$, with $\epsilon_{i} \sim \operatorname{Normal}\left(0, \sigma^{2}\right)$. We consider six simulation conditions. Conditions (1)(5) are equal to the conditions considered in Li and Lin (2010). In addition, condition (1) and (2) have also been considered in Kyung et al. (2010); Roy and Chakraborty (2016); Tibshirani (1996); Zou and Hastie (2005). Condition (6) has been included to investigate a setting in which $p>N$. The conditions are as follows ${ }^{6}$ :

1. $\boldsymbol{\beta}=(3,1.5,0,0,2,0,0,0)^{\prime} ; \sigma^{2}=9 ; \boldsymbol{X}$ generated from a multivariate normal distribution with mean vector $\mathbf{0}$, variances equal to 1 , and pairwise correlations between predictors equal to 0.5. The number of observations is $n=240$, with 40 observations for training and 200 observations for testing the model.

2. $\beta=(0.85,0.85,0.85,0.85,0.85,0.85,0.85,0.85)^{\prime}$; the other settings are equal to those in condition (1).

\footnotetext{
${ }^{6}$ We have also considered two additional conditions in which $p>n$ and the predictors are not highly correlated. Unfortunately, most shrinkage priors resulted in too much non-convergence to trust the results. A description of these additional conditions and the available results for the priors that did obtain enough convergence is available at https://osf.io/nveh3/. Additionally, we would like to refer to Kaseva (2018) where a more sparse, modified version of condition 1 is considered.
} 
3. $\beta=(\underbrace{3, \ldots, 3}_{15}, \underbrace{0, \ldots, 0}_{15}) ; \sigma^{2}=225 ; x_{j}=Z_{1}+\omega_{j}$, for $j=1, \ldots, 5$; $\boldsymbol{x}_{j}=Z_{2}+\omega_{j}$, for $j=6, \ldots, 10 ; \boldsymbol{x}_{j}=Z_{3}+\omega_{j}$, for $j=11, \ldots, 15$; and $\boldsymbol{x}_{j} \sim \operatorname{Normal}(0,1)$, for $j=16, \ldots, 30$. Here, $Z_{1}, Z_{2}$, and $Z_{3}$ are independent standard normal variables and $\omega_{j} \sim \operatorname{Normal}(0,0.01)$. The number of observations is $n=600$, with 200 observations for training and 400 observations for testing the model.

4. The number of observations is $n=800$, with 400 observations for training and 400 observations for testing the model; the other settings are equal to those in condition (3).

5. $\beta=(\underbrace{3, \ldots, 3}_{10}, \underbrace{0, \ldots, 0}_{10}, \underbrace{3, \ldots, 3}_{10})$; the number of observations is $n=440$, with 40 observations for training and 400 observations for testing the model; the other settings are equal to those in condition (3).

6. The number of observations is $n=55$, with 25 observations for training and 30 observations for testing the model; the other settings are equal to those in condition (5).

We simulate 500 data sets per condition. All Bayesian methods have been implemented in the software package Stan (Stan development team, 2017c), which we call from $R$ using Rstan (Stan development team, 2017a). We include the classical penalization methods available in the R-packages glmnet (Friedman et al., 2010) and grpreg (Breheny and Huang, 2015), i.e., the ridge, lasso, elastic net, and group lasso, for comparison. For the classical penalization methods, the penalty parameter $\lambda$ is selected based on cross-validation using 10 folds. We also include classical forward selection from the leaps (Lumley, 2017) package and we select the model based on three different criteria: the adjusted $R^{2}$, Mallows' $C_{p}$, and the BIC. For both the Bayesian and the classical group lasso, a grouping structure should be supplied for the analysis. We have used the grouping structure under which the data was simulated. Thus, for conditions 3 until 6 , we have four groups with the following regression coefficient belonging to each group: $G_{1}=$ $\beta_{1}, \ldots, \beta_{5}, G_{2}=\beta_{6}, \ldots, \beta_{10}, G_{3}=\beta_{11}, \ldots, \beta_{15}$, and $G_{4}=\beta_{16}, \ldots, \beta_{30}$. All code for the simulation study is available at https://osf.io/bf5up/.

\subsection{Outcomes}

The two main goals of regression analysis are: (1) to select variables that are relevant for predicting the outcome, and (2) to accurately predict the 


\section{BAYESian Penalization}

outcome. Therefore, we will focus on the performance of the shrinkage priors in terms of variable selection and prediction accuracy. Unlike frequentist penalization methods, Bayesian penalization methods do not automatically shrink regression coefficients to be exactly zero. A criterion is thus needed to select the relevant variables, for which we will use the credibility interval criterion. $^{7}$ Using the credibility interval criterion, a predictor is excluded when the credibility interval for $\beta_{j}$ covers 0 , and it is included when 0 is not contained in the credibility interval. This criterion thus depends on the percentage of posterior probability mass included in the credibility interval. We will investigate credibility intervals ranging from 0 to $100 \%$, with steps of $10 \%$. The optimal credibility interval is selected using the distance criterion (see e.g., Perkins and Schisterman, 2006), i.e.,

$$
\text { distance }=\sqrt{(1-\text { correct inclusion rate })^{2}+(\text { false inclusion rate })^{2}},
$$

The credibility interval with the lowest distance is optimal in terms of the highest correct inclusion rate and lowest false inclusion rate. For the selected credibility interval, we will report Matthews' correlation coefficient (MCC; Matthews, 1975), which is a measure indicating the quality of the classification. MCC ranges between -1 and +1 with $\mathrm{MCC}=-1$ indicating complete disagreement between the observed and predicted classifications and MCC $=+1$ indicating complete agreement.

To assess the prediction accuracy of the shrinkage priors, we will consider the prediction mean squared error (PMSE) for each replication. To compute the PMSE, we first estimate the regression coefficients $\hat{\beta}$ on the training data only. These estimates are then used to predict the responses on the outcome variable of the test set, $\boldsymbol{y}^{g e n}$, for which the actual responses, $\boldsymbol{y}$, are available. Prediction of $\boldsymbol{y}^{g e n}$ occurs within the "generated quantities" block in Stan, meaning that for each MCMC draw, $y_{i}^{\text {gen }}$ is generated such that we obtain the full posterior distribution for each $y_{i}^{g e n}$. The mean of this posterior distribution is used as estimate for $y_{i}^{\text {gen }}$. The PMSE for each replication can then be computed as: $\frac{1}{N} \sum_{i=1}^{N}\left(y_{i}^{g e n}-y_{i}\right)^{2}$. For each condition, this will result in 500 PMSEs, one for each replication, of which we will compute the median. Furthermore, to assess the uncertainty in the median PMSE estimate, we will bootstrap the standard error (SE) by resampling 500 PMSEs from the

\footnotetext{
${ }^{7}$ We have also considered the scaled neighborhood criterion ( $\mathrm{Li}$ and $\mathrm{Lin}, 2010$ ) and a fixed cut-off value to select the predictors. The scaled neighborhood criterion excludes a predictor if the posterior probability contained in $\left[-\sqrt{\operatorname{var}\left(\beta_{p} \mid \mathbf{y}\right)}, \sqrt{\operatorname{var}\left(\beta_{p} \mid \mathbf{y}\right)}\right]$ exceeds a certain threshold. However, this criterion generally performed worse than the credibility interval criterion. For the fixed cut-off value we excluded predictors when the posterior estimate $|\hat{\beta}| \leq 0.1$ based on Feng et al. (2015). However, the choice of this threshold is rather arbitrary and resulted in very high false inclusion rates.
} 


\section{BAYESIAN PENALizATion}

obtained PMSE values and computing the median. This process is repeated 500 times and the standard deviation of the 500 bootstrapped median PMSEs is used as SE of the median PMSE.

\subsection{Convergence}

Convergence will be assessed using split $\hat{R}$, which is a version of the often used potential scale reduction factor (PSRF; Gelman and Rubin, 1992) that is implemented in Stan (Stan development team, 2017b, p. 370-373). Additionally, Stan reports the number of divergent transitions. A divergent transition indicates that the approximation error in the algorithm accumulates (Betancourt, 2017; Monnahan et al., 2016), which can be caused by a too large step size, or because of strong curvature in the posterior distribution. As a result, it can be necessary to adapt the settings of the algorithm or to reparametrize the model. For the simulation, we initially employed a very small step size (0.001) and high target acceptance rate (0.999), however, these settings result in much slower sampling. Therefore, in the later conditions we used the default step size (1) and a lower target acceptance rate (0.85) and only reran the replications that did not converge with the stricter settings (i.e., smaller step size and higher target acceptance rate). Only if all parameters had a PSRF $<1.1$ and there were no divergent transitions, did we consider a replication as converged. ${ }^{8}$ We have only included those conditions in the results with at least $50 \%$ convergence (i.e., at least 250 converged replications). The convergence rates are available at https://osf.io/nveh3/.

\subsection{Prediction accuracy}

Table 2 shows the median PMSE per condition for the shrinkage priors and classical penalization methods. For the regularized horseshoe, the prior guess for the number of relevant variables $p_{0}$ was based on the data-generating model, however, the results were comparable when no prior guess or an incorrect prior guess was used. For all methods, the median PMSE increases as the condition becomes more complex. The smallest median PMSE per

\footnotetext{
${ }^{8}$ For the horseshoe prior, all replications in all conditions resulted in one or more divergent transitions, despite reparametrization of the model. The regularized horseshoe also resulted in divergent transitions for most replications, although the percentage of divergent transitions was on average much lower for the regularized horseshoe compared to the horseshoe. The percentages divergent transitions are available at https://osf.io/nveh3/. To be able to include these priors in the overview, we have only considered the PSRF to assess convergence and manually checked the traceplots. However, see Kaseva (2018) for a deeper investigation into the divergent transitions and alternative parametrizations of the horseshoe prior.
} 
Bayesian Penalization

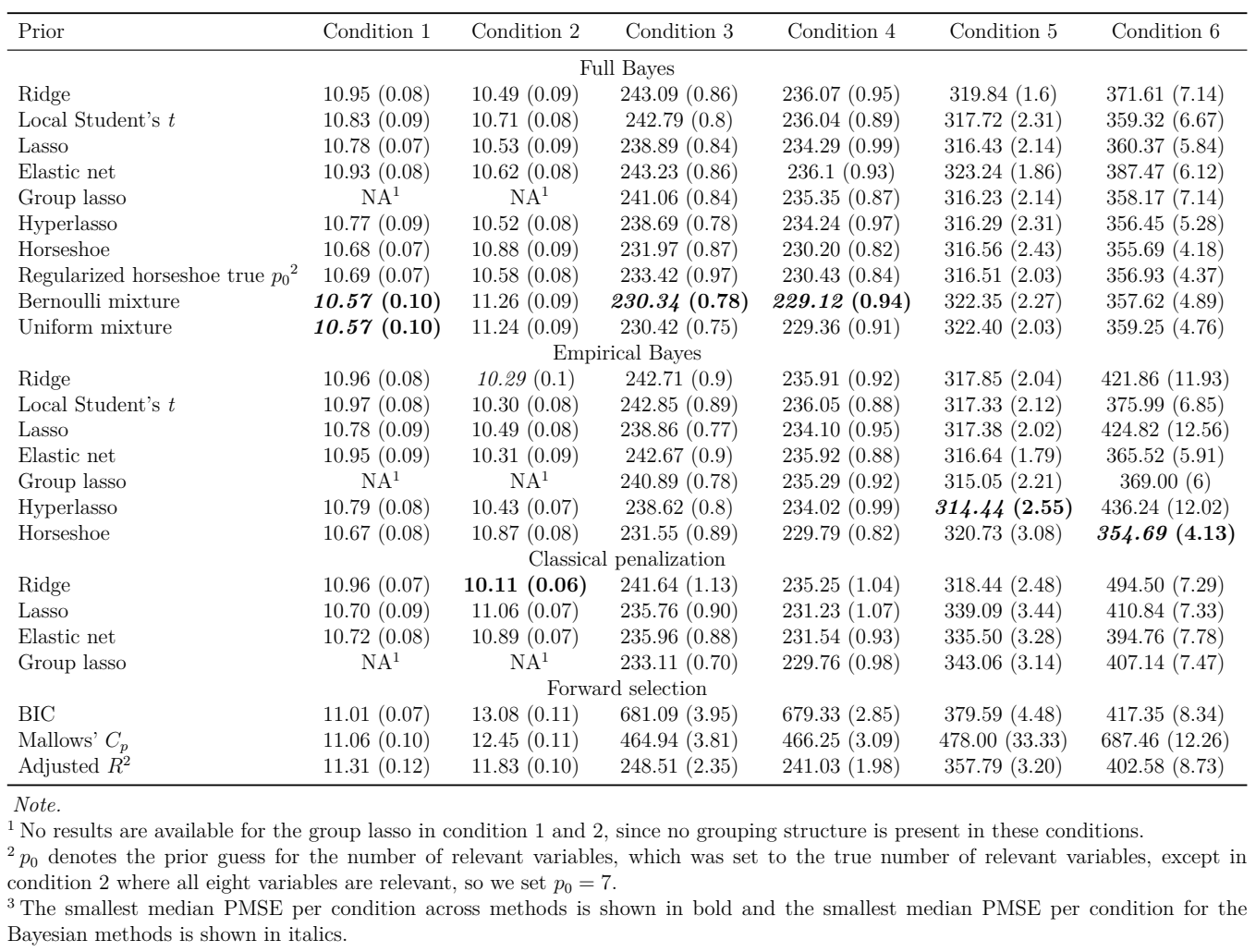

Table 2: Median prediction mean squared error (PMSE) with bootstrapped standard errors in brackets for the shrinkage priors.

condition across methods is shown in bold and the smallest median PMSE per condition for the Bayesian methods is shown in italics. In condition 1, 3, and 4 the full Bayesian Bernoulli mixture prior performs best; in condition 2, the classical ridge performs best; in condition 5, the empirical Bayesian hyperlasso performs best; and in condition 6, the empirical Bayesian horseshoe performs best. However, the differences between the methods are relatively small. Only in condition 6, where the number of predictors is larger than the number of observations, the differences between the methods in terms of PMSE become more pronounced. As expected, forward selection performs the worst, especially when Mallows' $C_{p}$ or the BIC is used to select the best model. This illustrates the advantage of using penalization, even when $p<n$. Overall, we can conclude that in terms of prediction accuracy the penalization methods perform quite similarly, except when $p>n .{ }^{9}$

\footnotetext{
${ }^{9}$ We have also computed the PMSE for a large test set with 1,000,000 observations as an approximation to the theoretical prediction error. In general, the theoretical PMSEs did not differ substantially from the PMSE in Table 2, except in condition 6 where the
} 
BAyESIAN PENALIZATION

\subsection{Variable selection accuracy}

theoretical PMSE was generally larger. The theoretical PMSEs are available online at https://osf.io/nveh3/ 


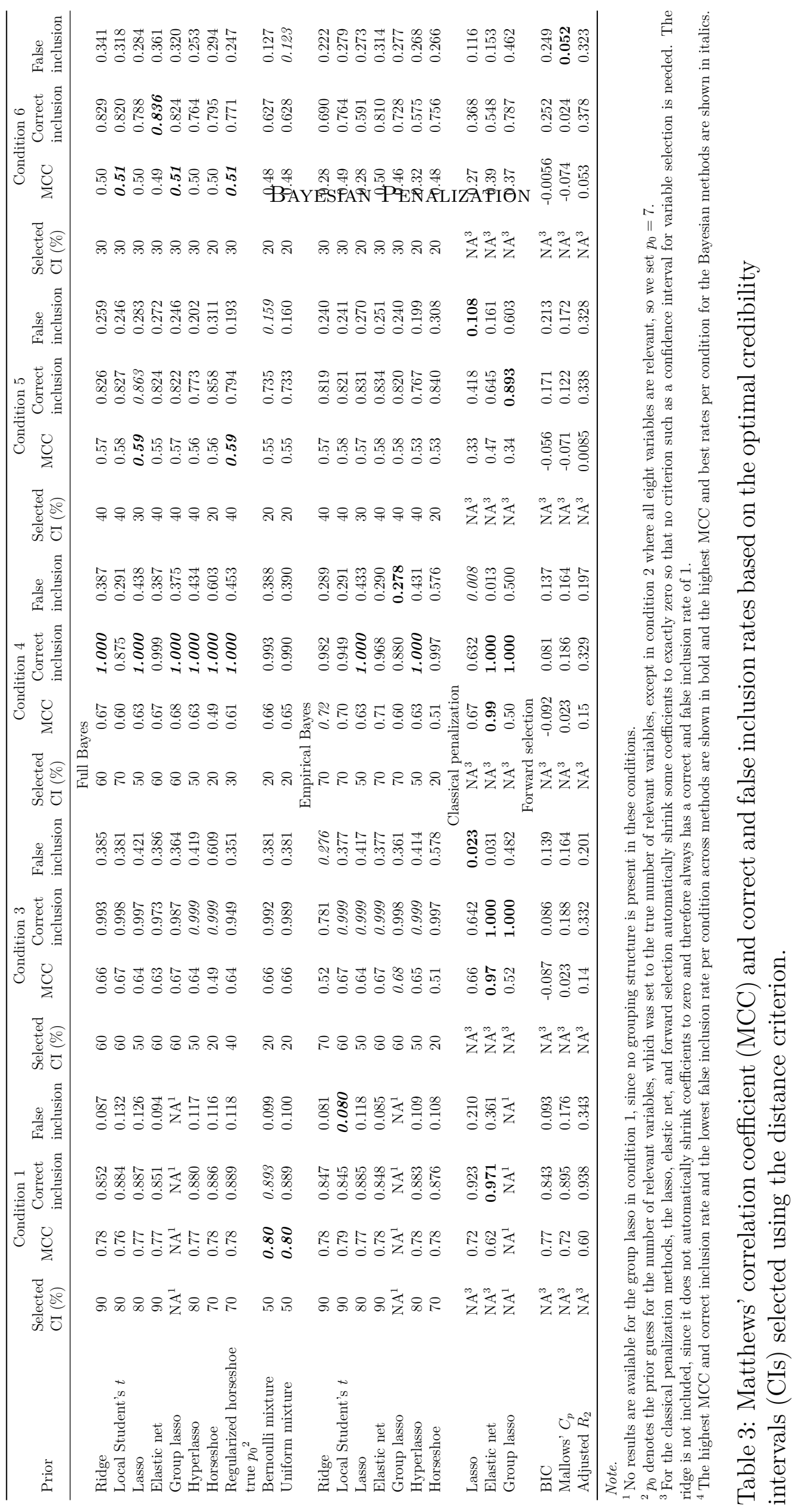




\section{BAYESIAN PENALizATion}

Table 3 shows MCC and the correct and false inclusion rates for the optimal CIs for the shrinkage priors and MCC and the inclusion rates for the classical penalization methods, which automatically select predictors. The bold values indicate the best inclusion rates across all methods, whereas the italic values indicate the best inclusion rates across the Bayesian methods. Again, for the regularized horseshoe the results were comparable regardless of whether a correct, incorrect, or no prior guess was used. In the first condition, the classical penalization methods outperform the Bayesian methods in terms of correct inclusion rates, but at the cost of higher false inclusion rates. This is a well known problem of the lasso and elastic net when cross-validation is used to select the penalty parameter $\lambda$. A solution to this problem is to use stability selection to determine $\lambda$ (Meinshausen and Bühlmann, 2010). The optimal Bayesian methods in the first condition based on the highest value for MCC are the mixture priors, both of which have reasonable correct and false inclusion rates. Note that, generally, the differences with the other Bayesian methods are relatively small in condition 1. In condition 3 and 4, the correct inclusion rates are generally high and the false inclusion rates are increased as well. As a result, the optimal Bayesian methods in condition 3 show a trade-off between correct and false inclusion rates, with the empirical Bayes group lasso having the highest value for MCC. However, the differences in MCC between most Bayesian methods are small and MCC is generally lower compared to condition 1 due to the increased false inclusion rates. In condition 4 , multiple methods show a correct inclusion rate of 1 , combined with a high false inclusion rate. In terms of MCC, the empirical Bayes ridge prior performs best. In condition 5 , both rates and thus the MCC values are slightly lower across all methods, which is a result of the optimal CI being smaller. The full Bayes lasso and regularized horseshoe perform best in terms of MCC, although the other shrinkage priors show comparable MCC values. Condition 6 shows the most pronounced differences between the methods and the greatest trade-off between correct and false inclusion rates. None of the Bayesian methods attain a value for the MCC greater than 0.51, and some shrinkage priors (i.e., the empirical Bayes ridge and lasso) result in a MCC value of only 0.28 . In conclusion, although there exist differences between the methods in terms of variable selection accuracy, there is not one method that performs substantially better than the other methods in terms of both correct and false inclusion rates. 


\section{BAyesian Penalization}

\section{Empirical applications}

We will now illustrate the shrinkage priors on two empirical data sets. An $\mathrm{R}$ package bayesreg is available online (https://github.com/sara-vanerp/bayesreg) that can be used to apply the shrinkage priors. The first illustration (math performance) shows the benefits of using shrinkage priors in a situation where the number of predictors is smaller than the number of observations. In the second illustration (communities and crime), the number of predictors is larger than the number of observations, and it is necessary to use some form of regularization in order to fit the model.

\subsection{Math performance}

In this illustration, we aim to predict the final math grade of 395 Portuegese students in secondary schools (Cortez and Silva, 2008), obtained from the UCL machine learning repository ${ }^{10}$ (Lichman, 2013). The data set includes 30 predictors covering demographic, social and school related characteristics, such as parents' education and the time spent studying. The continuous predictors were standardized and dummy variables were used for the categorical predictors, resulting in a total of 39 predictors. We split the data into an approximately equal training $(n=197)$ and test $(n=198)$ set.

Table 4 presents the computation time in seconds for each method, the prediction mean squared error, and the number of included predictors. We have not included the results for the horseshoe prior because this prior resulted in divergent transitions, which in turn led to instable results (specifically, the PMSE varied greatly when rerunning the analysis).

It is clear that the Bayesian methods are computationally much more intensive than the classical penalization methods, especially the regularized horseshoe and mixture priors. The advantage brought by this increased computation time, however, is the more straightforward interpretation of results such as credibility intervals and the automatic computation of uncertainty estimates. This can be seen in Figure 7 which shows the posterior density for one regression coefficient $\beta_{1}$ using the lasso prior and its $95 \%$ credibility interval (i.e., the shaded dark blue area). The bootstrapped 95\% confidence interval obtained using the HDCI package (Liu et al., 2017) in R is shown by the dashed grey lines and can be seen to underestimate the uncertainty. This problem is often observed using classical lasso estimation (Kyung et al., 2010). The PMSE clearly illustrates the advantage of penalization, even

\footnotetext{
${ }^{10}$ The data is available at https://archive.ics.uci.edu/ml/datasets/Student+Performance
} 
Bayesian Penalization

\begin{tabular}{lccc}
\hline Shrinkage prior & Computation time (seconds) & PMSE & Number of included predictors \\
\hline & 179 & & \\
Ridge & 361 & 19.53 & 22 \\
Local Student's $t$ & 219 & 19.44 & 22 \\
Lasso & 354 & 19.25 & 22 \\
Elastic net & 342 & 19.53 & 23 \\
Group lasso & 199 & 19.36 & 22 \\
Hyperlasso & 1474 & 19.18 & 19 \\
Regularized horseshoe with $p_{0}$ & 24524 & 19.12 & 17 \\
Bernoulli mixture & 4370 & 19.31 & 9 \\
Uniform mixture & Empirical Bayes & 19.28 & \\
& 341 & 19.42 & 22 \\
Ridge & 443 & 19.47 & 22 \\
Local Student's $t$ & 444 & 19.26 & 22 \\
Lasso & 603 & 19.41 & 22 \\
Elastic net & 534 & 19.50 & 19 \\
Group lasso & 387 & 19.11 & 39 \\
Hyperlasso & Classical penalization & & 38 \\
& 0.013 & 22.56 & 20 \\
Ordinary least squares $($ OLS) & 0.118 & 18.95 & 20 \\
Ridge & 0.0 .072 & 19.11 & 21 \\
Lasso & 0.0 .053 & 19.25 & 43 \\
Elastic net & 0.187 & 19.43 & 25 \\
Group lasso & Forward selection & & \\
& 0.006 & 21.13 & 21.42 \\
BIC & 0.006 & 22.44 & \\
Mallows' $C_{p}$ & 0.006 & & \\
Adjusted $R^{2}$ & & & \\
\hline & & & \\
\hline
\end{tabular}

Table 4: Computation time in seconds (with a $2.8 \mathrm{GHz}$ Intel Core i7 processor), prediction mean squared error (PMSE), and number of included predictors for the different methods for the math performance application

though the number of predictors is not greater than the sample size. Compared to regression using OLS, all penalization methods show lower PMSEs. Moreover, all penalization methods outperform forward selection in terms of PMSE. Between the different penalization methods, differences in PMSE are small. 


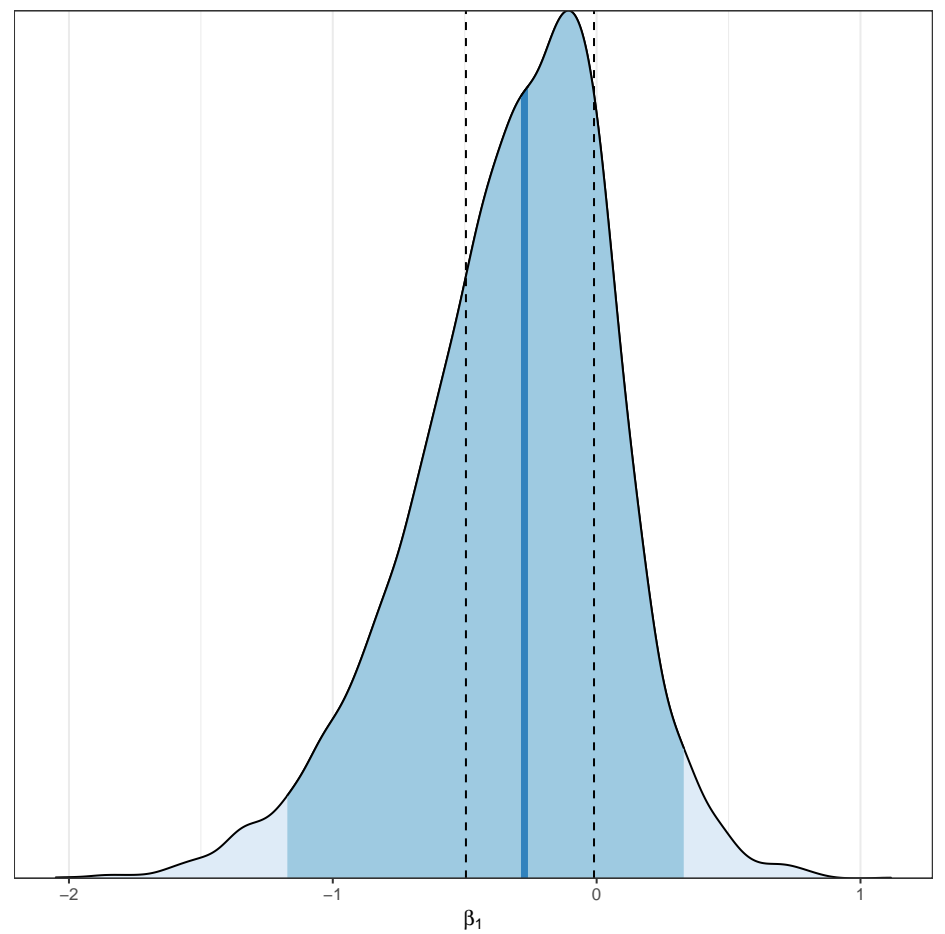

Figure 7: Posterior density for $\beta_{1}$ in the math performance application using the Bayesian lasso. The dark blue line depicts the posterior median, the shaded dark blue area depicts the $95 \%$ credibility interval. The black dashed lines depict the bootstrapped $95 \%$ confidence interval of the classical lasso. 


\section{BAyESian PENALIZATION}

The last column in Table 4 reports the number of included predictors for each method. Figure 8 shows which predictors are included for each method. Each point indicates an included predictor, based on the optimal CI from condition 5 in the simulation study. OLS does not exclude any predictors, neither does the classical ridge generally although in this data set one coefficient was estimated to be zero. Most shrinkage priors included 22 predictors, with the hyperlasso and regularized horseshoe resulting in a slightly sparser solution. The mixture priors selected much less predictors (9) compared to the other methods. The number of included predictors for the forward selection method ranged from 4 to 25, depending on the criterium used to select the best model.

Based on the predicted errors and the number of included predictors, we conclude that essentially all Bayesian methods and the classical penalization methods performed best. The computation time for the Bayesian methods was considerably larger than for the classical methods. However, this increased computation time results in automatic availability of uncertainty estimates which were generally larger compared to classical bootstrapped confidence intervals.

\subsection{Communities and crime}

We illustrate the shrinkage priors on a data set containing 125 predictors of the number of violent crimes per 100,000 residents in different communities in the US (Redmond and Baveja, 2002) obtained from the UCL machine learning repository ${ }^{11}$ (Lichman, 2013). The predictor variables include community characteristics, such as the median family income and the percentage of housing that is occupied, as well as law enforcement characteristics, such as the number of police officers and the police operating budget. We created dummy variables for the two nominal predictors in the data set, resulting in a total of 172 predictors. For the group lasso, all dummy variables corresponding to one predictor make up a group. The number of observations is 319, after removing all cases with at least one missing value on any of the predictors. $^{12}$ We split the data into approximately equal training $(n=159)$ and test $(n=160)$ sets. All predictors were normalized to have zero mean and unit variance and the outcome variable was log transformed.

Table 5 reports the computation time in seconds for each method, as

\footnotetext{
${ }^{11}$ We used the unnormalized data, available at https://archive.ics.uci.edu/ml/datasets/Communities+and+Crime+Unnormalized

${ }^{12}$ Although the Bayesian framework allows for straightforward imputation of missing values, we removed all cases with missing values to provide an illustration of the shrinkage methods in a sparse data set.
} 


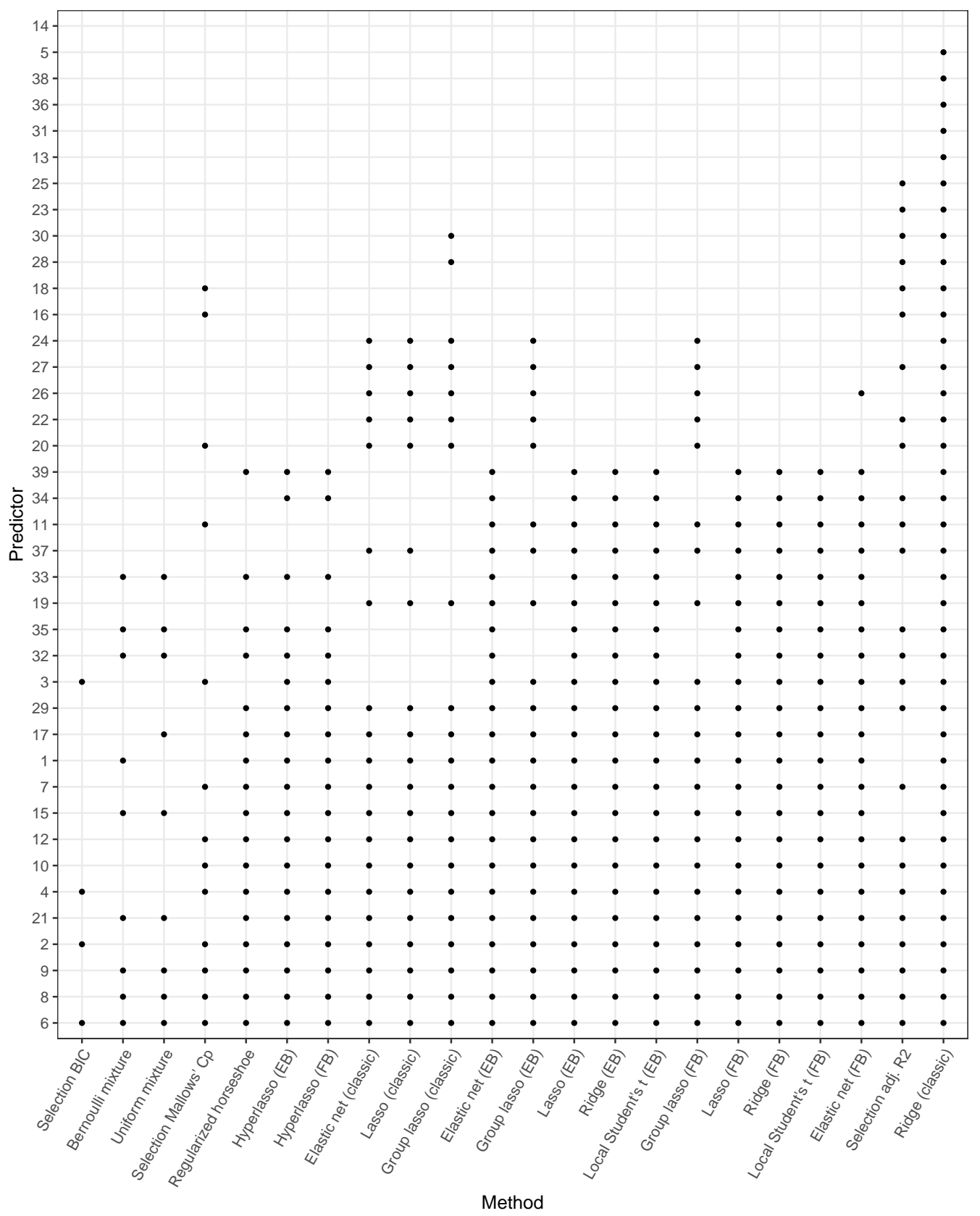

Figure 8: Overview of the included predictors for each method in the math performance application. Points indicate that a predictor is included based on the optimal credibility interval (CI) from condition 5 in the simulation study. The methods on the $\mathrm{x}$-axis are ordered such that the method that includes the least predictors is on the left and the method that includes the most predictors is on the right. The predictors on the y-axis are ordered with the predictor being included the least on top and the predictor being included the most at the bottom. 


\section{BAYESIAN PENALIZATION}

well as the PMSE and the number of selected variables. Again, the horseshoe prior resulted in divergent transitions and is therefore excluded from the results. The posterior density using the lasso prior for $\beta_{15}$ is shown in Figure 9, with the dark blue shaded area depicting the $95 \%$ credibility intervals and the dashed black lines depicting the bootstrapped 95\% confidence interval of the classical lasso. Again, the bootstrapped confidence interval is much smaller than the Bayesian credibility interval and located far from the posterior median estimate (i.e., the dark blue line).

\begin{tabular}{lccc}
\hline Shrinkage prior & Computation time (seconds) & PMSE & Number of included predictors \\
\hline & 677 Bayes & & \\
Ridge & 1973 & 0.217 & 61 \\
Local Student's $t$ & 2068 & 0.216 & 60 \\
Lasso & 242 & 0.216 & 46 \\
Elastic net & 3044 & 0.216 & 62 \\
Group lasso & 1066 & 0.216 & 61 \\
Hyperlasso & 15803 & 0.215 & 46 \\
Regularized horseshoe with $p_{0}$ & 60006 & 0.226 & 31 \\
Bernoulli mixture & 26080 & 1.706 & 54 \\
Uniform mixture & Empirical Bayes & 1.683 & 54 \\
& 1195 & 0.218 & 60 \\
Ridge & 1912 & 0.216 & 57 \\
Local Student's $t$ & 4207 & 0.215 & 46 \\
Lasso & 417 & 0.217 & 57 \\
Elastic net & 3992 & 0.217 & 62 \\
Group lasso & 2016 & 0.215 & 46 \\
Hyperlasso & Classical penalization & \\
Ridge & 0.376 & 0.258 & 160 \\
Lasso & 0.200 & 0.508 & 33 \\
Elastic net & 0.164 & 0.460 & 26 \\
Group lasso & 0.408 & 0.663 & 55 \\
BIC & Forward selection & & 17 \\
Mallows' $C_{p}$ & 0.023 & 1.500 & 141 \\
Adjusted $R^{2}$ & 0.023 & 0.276 & \\
\hline & 0.023 & 4.093 & \\
\hline
\end{tabular}

Table 5: Computation time in seconds (with a $2.8 \mathrm{GHz}$ Intel Core i7 processor), prediction mean squared error (PMSE), and number of included predictors for the different methods for the crime application

In addition, most Bayesian methods resulted in a lower PMSE than the classical methods, except for the mixture priors. The forward selection method resulted in much larger PMSEs, except when Mallows' $C_{p}$ was used to find the best model, however, this model retained only 1 predictor. On the other hand, using the Adjusted $R^{2}$ criterion led to a model that included 141 predictors. This illustrates the arbitrariness of using forward selection. Figure 10 shows which predictors are included for each method. Each point 
indicates an included predictor, based on the optimal CI from condition 6 in the simulation study. Apart from the forward selection methods, the classical elastic net excludes most predictors. Interestingly, the Bayesian elastic net and lasso retain many more predictors than the classical elastic net and lasso. However, not all predictors that are retained by the classical lasso and elastic net are also retained by the Bayesian lasso and elastic net. Specifically, the predictors included by the classical methods but not by the Bayesian methods all correspond to dummy variables for State. The hyperlasso and lasso methods all include 46 predictors, whereas the ridge, local Student's $t$, elastic net, and group lasso priors all retain around 60 predictors. The mixture priors both include 54 predictors. The regularized horseshoe retains the least predictors of all Bayesian methods, only 31. The classical ridge retains almost all predictors, but estimated some coefficients to be equal to zero in this data set.

Based on this illustration, we conclude that the Bayesian penalization methods outperform the classical penalization methods in terms of prediction error. The prediction errors of the Bayesian penalization methods do not differ substantially, except for the mixture priors which showed larger PMSEs. The shrinkage priors differ in how much shrinkage they perform and thus in the number of predictors that are selected. 


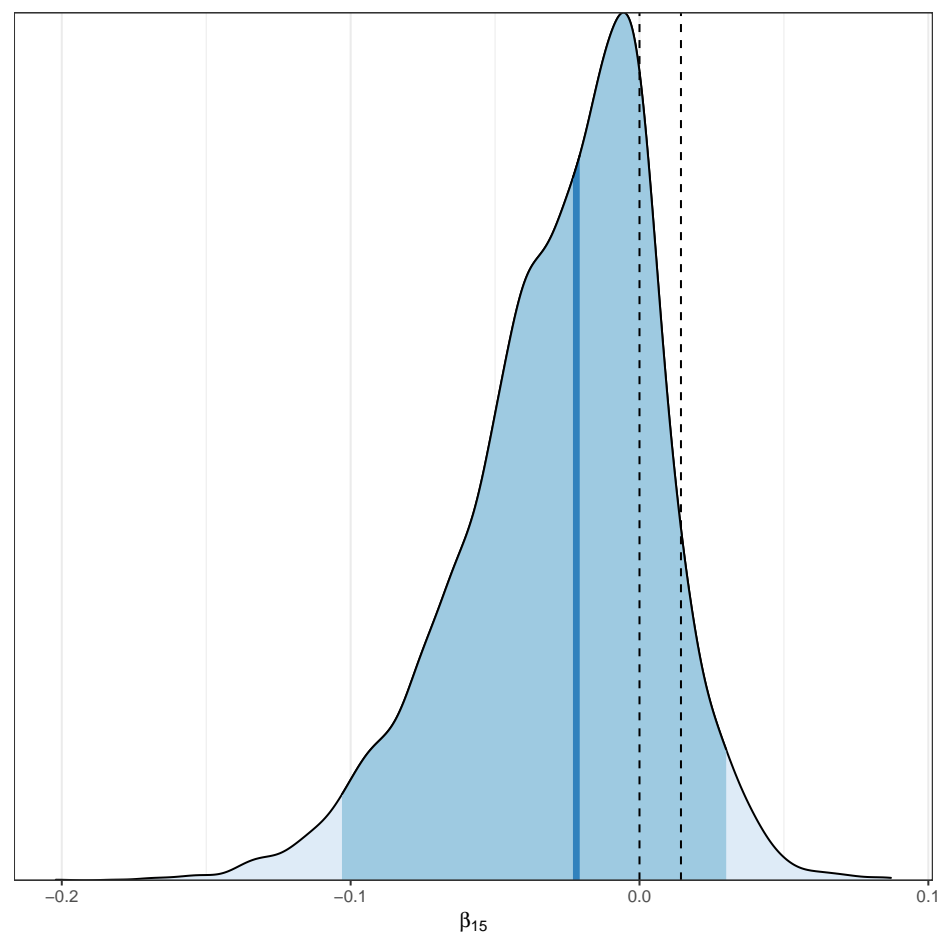

Figure 9: Posterior density for $\beta_{15}$ in the crime application using the Bayesian lasso. The dark blue line depicts the posterior median, the shaded dark blue area depicts the $95 \%$ credibility interval. The black dashed lines depict the bootstrapped $95 \%$ confidence interval of the classical lasso. 


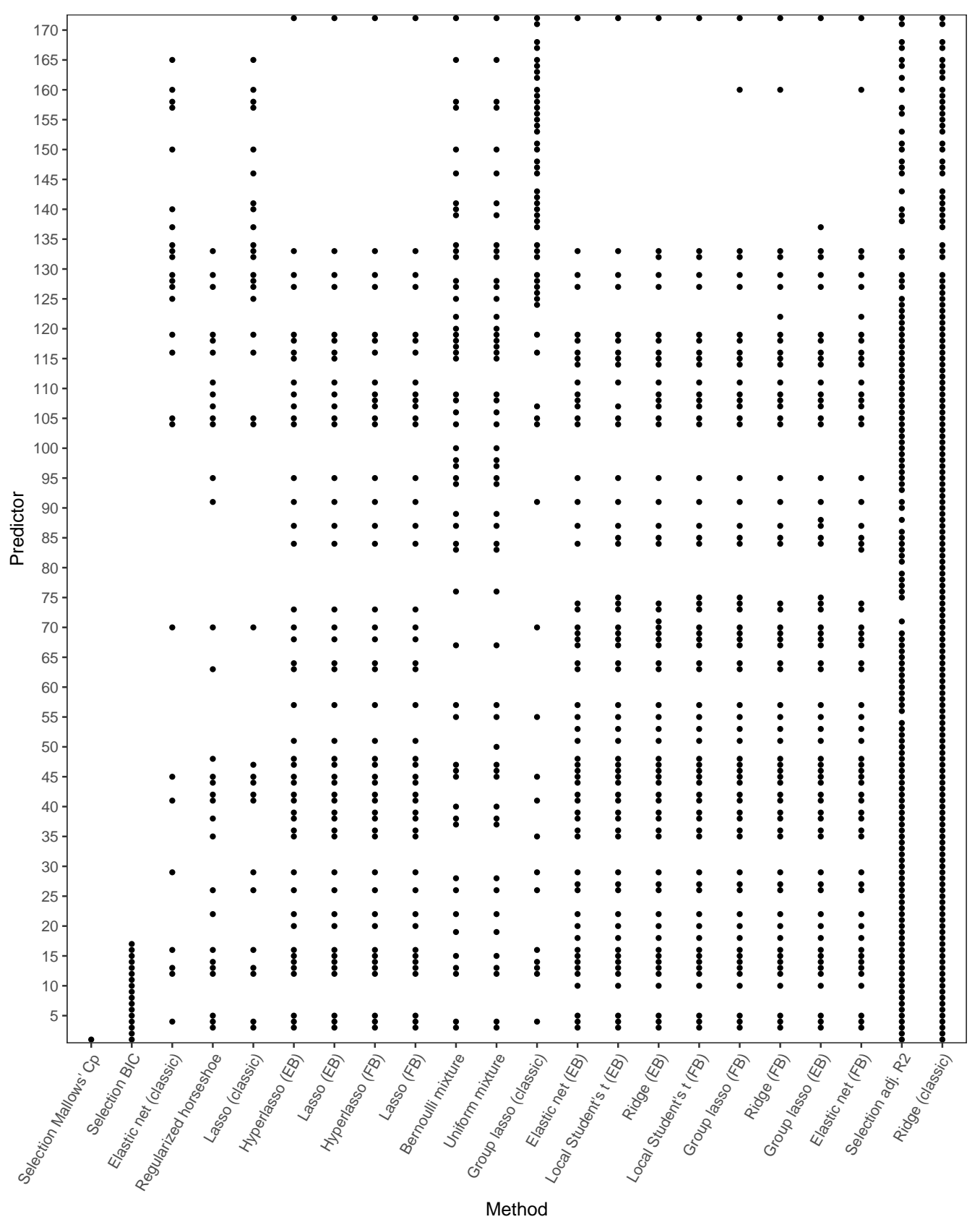

Figure 10: Overview of the included predictors for each method in the crime application. Points indicate that a predictor is included based on the optimal credibility interval (CI) from condition 6 in the simulation study. The methods on the $\mathrm{x}$-axis are ordered such that the method that includes the least predictors is on the left and the method that includes the most predictors is on the right. 


\section{BAyesian Penalization}

\section{Discussion}

The aim of this paper was to provide insights about the different shrinkage priors that have been proposed for Bayesian penalization to avoid overfitting of regression models in the case of many predictors. We have reviewed the literature on shrinkage priors and presented them in a general framework of scale mixtures of normal distributions to enable theoretical comparisons between the priors. To model the penalty parameter $\lambda$, which is a central part of the penalized regression model, a full Bayes and an empirical Bayes approach were employed.

Although the various prior distributions differ substantially from each other, e.g., regarding their tails or convexity, the priors performed very similarly in the simulation study in those conditions where $p<n$. Overall, the performance was comparable to the classical penalization approaches. The math performance example clearly showed the advantage of using penalization to avoid overfitting when $p<n$. As in the simulation study, the prediction errors in the math example were comparable across penalization methods, although the number of included predictors varied across methods. Finally, although classical penalization is much faster than Bayesian penalization, it does not automatically provide accurate uncertainty estimates and the bootstrapped confidence intervals obtained for the classical methods were generally much smaller compared to the Bayesian credibility intervals.

The differences between the methods became more pronounced when $p>n$. In condition 6 of the simulation study, the (regularized) horseshoe and hyperlasso priors performed substantially better then most of the other shrinkage priors in terms of PMSE. This is most likely due to the fact that the hyperlasso and (regularized) horseshoe are non-convex global-local shrinkage priors and are therefore particularly adept at keeping large coefficients large, while shrinking the small coefficients enough towards zero. Future research should consider various high-dimensional simulation conditions to further explore the performance of the shrinkage priors in such settings, for example by varying the correlations between the predictors. The crime example illustrated the use of the penalization methods further in a $p>n$ situation. In this example, most Bayesian approaches resulted in smaller prediction errors than the classical approaches (except for the mixture priors). Also in terms of the predictors that were included there were considerable differences between the various approaches.

An important goal of the shrinkage methods discussed in this paper is the ultimate selection of relevant variables. Throughout this paper, we have focused on the use of marginal credibility intervals to do so. However, the use of marginal credibility intervals to perform variable selection can be prob- 


\section{BAYESIAN PENALIZATION}

lematic, since the marginal intervals can behave differently compared to joint credibility intervals. This is especially the case for global shrinkage priors, such as the (regularized) horseshoe prior since these priors induce shrinkage on all variables jointly (Piironen et al., 2017). Future research should investigate whether the variable selection accuracy can be further improved by using methods that jointly select relevant variables (for example, projection predictive variable selection; Piironen and Vehtari, 2016, or decoupled shrinkage and selection; Hahn and Carvalho, 2015).

Throughout this paper, we focused on the linear regression model. Hopefully, the results presented in this paper and the corresponding $\mathrm{R}$ package bayesreg available at https://github.com/sara-vanerp/bayesreg will lead to an increased use of penalization methods in psychology, because of the improved performance in terms of prediction error and variable selection accuracy compared to forward subset selection. The shrinkage priors investigated here can be applied in more complex models in a straightforward manner. For example, in generalized linear regression models such as logistic and Poisson regression models, the only necessary adaptation is to incorporate a link function in the model. Although not currently available in the Rpackage, the available Stan modelfiles can be easily adapted to generalized linear models (GLMs). Additionally, packages such as brms (Bürkner, 2017) and rstanarm (Stan Development Team, 2016) include several of the shrinkage priors described here, or allow the user to specify them manually. Both packages support (multilevel) GLMs, although rstanarm relies on precompiled models and is therefore less flexible than brms. Currently, an active area of research employs Bayesian penalization in latent variable models, such as factor models (see e.g., Lu et al., 2016; Jacobucci and Grimm, 2018) and quantile structural equation models (see e.g., Feng et al., 2017). The characteristics and behaviors of the shrinkage priors presented in this paper can be a useful first step in solving these more challenging problems. 


\section{Acknowledgements}

This research was supported by a Research Talent Grant from the Netherlands Organisation for Scientific Research. We would like to thank Aki Vehtari, Carlos Carvalho, Tuomas Kaseva, and Charlie Strauss for providing helpful comments on an earlier version of this manuscript and pointing out relevant references.

\section{References}

Alhamzawi, R., Yu, K., and Benoit, D. F. (2012). Bayesian adaptive lasso quantile regression. Statistical Modelling, 12(3):279-297.

Andersen, M. R., Vehtari, A., Winther, O., and Hansen, L. K. (2017). Bayesian inference for spatio-temporal spike-and-slab priors. Journal of Machine Learning Research, 18(139):1-58.

Armagan, A., Dunson, D. B., and Lee, J. (2013). Generalized double pareto shrinkage. Statistica Sinica.

Azmak, O., Bayer, H., Caplin, A., Chun, M., Glimcher, P., Koonin, S., and Patrinos, A. (2015). Using big data to understand the human condition: The kavli HUMAN project. Big Data, 3(3):173-188.

Bae, K. and Mallick, B. K. (2004). Gene selection using a two-level hierarchical bayesian model. Bioinformatics, 20(18):3423-3430.

Berger, J. O. (2006). The case for objective bayesian analysis. Bayesian Analysis, 3:385-402.

Betancourt, M. (2017). A conceptual introduction to hamiltonian monte carlo. arXiv preprint arXiv:1701.02434.

Bhadra, A., Datta, J., Polson, N. G., and Willard, B. (2016). The horseshoe+ estimator of ultra-sparse signals. Bayesian Analysis.

Bhadra, A., Datta, J., Polson, N. G., and Willard, B. T. (2017). Lasso meets horseshoe: A survey. arXiv preprint arXiv:1706.10179.

Bhattacharya, A., Pati, D., Pillai, N. S., and Dunson, D. B. (2012). Bayesian shrinkage. arXiv preprint arXiv:1212.6088.

Bornn, L., Gottardo, R., and Doucet, A. (2010). Grouping priors and the Bayesian elastic net. arXiv preprint arXiv:1001.4083. 


\section{BAYESian Penalization}

Breheny, P. and Huang, J. (2015). Group descent algorithms for nonconvex penalized linear and logistic regression models with grouped predictors. Statistics and Computing, 25:173-187.

Bürkner, P.-C. (2017). brms: An R package for Bayesian multilevel models using Stan. Journal of Statistical Software, 80(1):1-28.

Caron, F. and Doucet, A. (2008). Sparse bayesian nonparametric regression. In Proceedings of the 25th international conference on Machine learning ICML '08. Association for Computing Machinery (ACM).

Carvalho, C. M., Polson, N. G., and Scott, J. G. (2010). The horseshoe estimator for sparse signals. Biometrika, 97(2):465-480.

Cortez, P. and Silva, A. M. G. (2008). Using data mining to predict secondary school student performance. In In A. Brito and J. Teixeira Eds., Proceedings of 5th FUture BUsiness TEChnology Conference, pages 5-12.

Derksen, S. and Keselman, H. J. (1992). Backward, forward and stepwise automated subset selection algorithms: Frequency of obtaining authentic and noise variables. British Journal of Mathematical and Statistical Psychology, 45(2):265-282.

Fan, J. and Li, R. (2001). Variable selection via nonconcave penalized likelihood and its oracle properties. Journal of the American Statistical Association, 96(456):1348-1360.

Fawcett, T. (2015). Mining the quantified self: Personal knowledge discovery as a challenge for data science. Big Data, 3(4):249-266.

Feng, X.-N., Wang, Y., Lu, B., and Song, X.-Y. (2017). Bayesian regularized quantile structural equation models. Journal of Multivariate Analysis, 154:234-248.

Feng, X.-N., Wu, H.-T., and Song, X.-Y. (2015). Bayesian adaptive lasso for ordinal regression with latent variables. Sociological Methods $\&$ Research.

Friedman, J., Hastie, T., and Tibshirani, R. (2010). Regularization paths for generalized linear models via coordinate descent. Journal of Statistical Software, 33(1):1-22.

Gelman, A. (2006). Prior distributions for variance parameters in hierarchical models (comment on article by browne and draper). Bayesian Analysis, $1(3): 515-534$. 


\section{BAYESIAN PEnAlization}

Gelman, A. and Rubin, D. B. (1992). Inference from iterative simulation using multiple sequences. Statistical Science, 7(4):457-472.

George, E. I. and McCulloch, R. E. (1993). Variable selection via gibbs sampling. Journal of the American Statistical Association, 88(423):881.

Ghosh, J., Li, Y., and Mitra, R. (2017). On the use of cauchy prior distributions for bayesian logistic regression. Bayesian Analysis.

Griffin, J. and Brown, P. (2017). Hierarchical shrinkage priors for regression models. Bayesian Analysis, 12(1):135-159.

Griffin, J. E. and Brown, P. J. (2005). Alternative prior distributions for variable selection with very many more variables than observations. University of Warwick. Centre for Research in Statistical Methodology.

Griffin, J. E. and Brown, P. J. (2011). Bayesian hyper-lassos with non-convex penalization. Australian \&5 New Zealand Journal of Statistics, 53(4):423442.

Hahn, P. R. and Carvalho, C. M. (2015). Decoupling shrinkage and selection in bayesian linear models: A posterior summary perspective. Journal of the American Statistical Association, 110(509):435-448.

Hans, C. (2009). Bayesian lasso regression. Biometrika, 96(4):835-845.

Hastie, T., Tibshirani, R., and Wainwright, M. (2015). Statistical learning with sparsity. CRC press.

Hoerl, A. E. and Kennard, R. W. (1970). Ridge regression: Biased estimation for nonorthogonal problems. Technometrics, 12(1):55-67.

Hsiang, T. C. (1975). A bayesian view on ridge regression. The Statistician, 24(4):267.

Ishwaran, H. and Rao, J. S. (2005). Spike and slab variable selection: Frequentist and bayesian strategies. The Annals of Statistics, 33(2):730-773.

Jacobucci, R. and Grimm, K. J. (2018). Comparison of frequentist and bayesian regularization in structural equation modeling. Structural Equation Modeling: A Multidisciplinary Journal, pages 1-11.

Kaseva, T. (2018). Convergence diagnosis and comparison of shrinkage priors. Github repository. 


\section{Bayesian Penalization}

Kyung, M., Gill, J., Ghosh, M., and Casella, G. (2010). Penalized regression, standard errors, and bayesian lassos. Bayesian Analysis, 5(2):369-411.

Li, Q. and Lin, N. (2010). The bayesian elastic net. Bayesian Analysis, $5(1): 151-170$.

Lichman, M. (2013). UCI machine learning repository.

Liu, H., Xu, X., and Li, J. J. (2017). HDCI: High Dimensional Confidence Interval Based on Lasso and Bootstrap. R package version 1.0-2.

Lu, Z.-H., Chow, S.-M., and Loken, E. (2016). Bayesian factor analysis as a variable-selection problem: Alternative priors and consequences. Multivariate Behavioral Research, 51(4):519-539.

Lumley, T. (2017). leaps: Regression Subset Selection. R package version 3.0 .

Matthews, B. (1975). Comparison of the predicted and observed secondary structure of t4 phage lysozyme. Biochimica et Biophysica Acta (BBA) Protein Structure, 405(2):442-451.

McNeish, D. M. (2015). Using lasso for predictor selection and to assuage overfitting: A method long overlooked in behavioral sciences. Multivariate Behavioral Research, 50(5):471-484.

Meinshausen, N. and Bühlmann, P. (2010). Stability selection. Journal of the Royal Statistical Society: Series B (Statistical Methodology), 72(4):417473.

Meuwissen, T. H., Hayes, B. J., and Goddard, M. E. (2001). Prediction of total genetic value using genome-wide dense marker maps. Genetics, 157(4):1819-1829.

Mitchell, T. J. and Beauchamp, J. J. (1988). Bayesian variable selection in linear regression. Journal of the American Statistical Association, 83(404):1023-1032.

Monnahan, C. C., Thorson, J. T., and Branch, T. A. (2016). Faster estimation of bayesian models in ecology using hamiltonian monte carlo. Methods in Ecology and Evolution, 8(3):339-348.

Mulder, J. and Pericchi, L. R. (2018). The matrix-f prior for estimating and testing covariance matrices. Bayesian Analysis, 13(4):1189-1210. 


\section{BAYESIAN PENALIZATION}

Park, T. and Casella, G. (2008). The bayesian lasso. Journal of the American Statistical Association, 103(482):681-686.

Peltola, T., Havulinna, A. S., Salomaa, V., and Vehtari, A. (2014). Hierarchical bayesian survival analysis and projective covariate selection in cardiovascular event risk prediction. In Proceedings of the Eleventh UAI Conference on Bayesian Modeling Applications Workshop-Volume 1218, pages 79-88. CEUR-WS. org.

Perkins, N. J. and Schisterman, E. F. (2006). The inconsistency of "optimal" cutpoints obtained using two criteria based on the receiver operating characteristic curve. American Journal of Epidemiology, 163(7):670-675.

Piironen, J., Betancourt, M., Simpson, D., and Vehtari, A. (2017). Contributed comment on article by van der Pas, Szabó, and van der Vaart. Bayesian Analysis, 12(4):1264-1266.

Piironen, J. and Vehtari, A. (2016). Comparison of bayesian predictive methods for model selection. Statistics and Computing, 27(3):711-735.

Piironen, J. and Vehtari, A. (2017). Sparsity information and regularization in the horseshoe and other shrinkage priors. Electronic Journal of Statistics, 11(2):5018-5051.

Polson, N. G. and Scott, J. G. (2011). Shrink globally, act locally: Sparse bayesian regularization and prediction. In Bayesian Statistics 9, pages 501-538. Oxford University Press (OUP).

Polson, N. G. and Scott, J. G. (2012). On the half-cauchy prior for a global scale parameter. Bayesian Analysis, 7(4):887-902.

Polson, N. G., Scott, J. G., and Windle, J. (2014). The bayesian bridge. Journal of the Royal Statistical Society: Series B (Statistical Methodology), 76(4):713-733.

Redmond, M. and Baveja, A. (2002). A data-driven software tool for enabling cooperative information sharing among police departments. European Journal of Operational Research, 141(3):660-678.

Roy, V. and Chakraborty, S. (2016). Selection of tuning parameters, solution paths and standard errors for bayesian lassos. Bayesian Analysis.

Stan Development Team (2016). rstanarm: Bayesian applied regression modeling via Stan. R package version 2.13.1. 


\section{BAYESIAN PENALIZATION}

Stan development team (2017a). RStan: The R interface to Stan, R package version 2.16.2.

Stan development team (2017b). Stan Modeling Language Users Guide and Reference Manual, version 2.17.0.

Stan development team (2017c). The Stan Core Library, version 2.16.0.

Tibshirani, R. (1996). Regression shrinkage and selection via the lasso. Journal of the Royal Statistical Society. Series B (Methodological), pages 267288.

Tibshirani, R. (2011). Regression shrinkage and selection via the lasso: a retrospective. Journal of the Royal Statistical Society: Series B (Statistical Methodology), 73(3):273-282.

van de Wiel, M. A., Beest, D. E. t., and Münch, M. (2017). Learning from a lot: Empirical bayes in high-dimensional prediction settings. arXiv preprint arXiv:1709.04192.

van Erp, S., Mulder, J., and Oberski, D. L. (2018). Prior sensitivity analysis in default bayesian structural equation modeling. Psychological Methods, 23(2):363-388.

Vehtari, A., Gabry, J., Yao, Y., and Gelman, A. (2018). loo: Efficient leaveone-out cross-validation and waic for bayesian models. $\mathrm{R}$ package version 2.0.0.

West, M. (1987). On scale mixtures of normal distributions. Biometrika, pages 646-648.

Wolpert, D. H. and Strauss, C. E. M. (1996). What bayes has to say about the evidence procedure. In Maximum Entropy and Bayesian Methods, pages 61-78. Springer Netherlands.

Yuan, M. and Lin, Y. (2006). Model selection and estimation in regression with grouped variables. Journal of the Royal Statistical Society: Series B (Statistical Methodology), 68(1):49-67.

Zhao, S., Gao, C., Mukherjee, S., and Engelhardt, B. E. (2016). Bayesian group factor analysis with structured sparsity. Journal of Machine Learning Research, 17(196):1-47.

Zou, H. (2006). The adaptive lasso and its oracle properties. Journal of the American Statistical Association, 101(476):1418-1429. 


\section{BAYESIAN PENALizATION}

Zou, H. and Hastie, T. (2005). Regularization and variable selection via the elastic net. Journal of the Royal Statistical Society: Series B (Statistical Methodology), 67(2):301-320. 